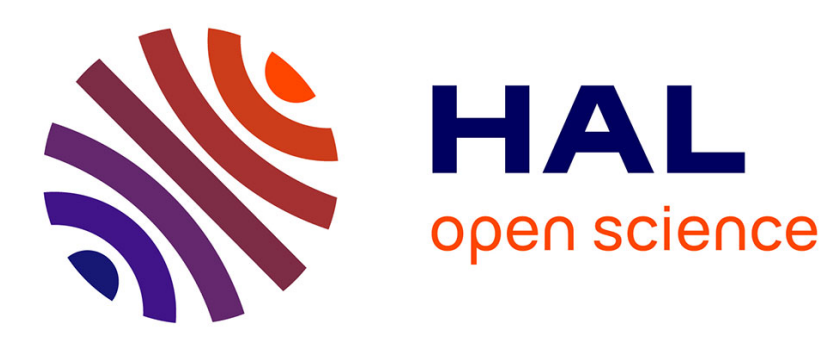

\title{
A space-discretized mixed-integer linear model for air-conflict resolution with speed and heading maneuvers \\ Jérémy Omer
}

\section{To cite this version:}

Jérémy Omer. A space-discretized mixed-integer linear model for air-conflict resolution with speed and heading maneuvers. Computers and Operations Research, 2015, 58, pp.75-86. 10.1016/j.cor.2014.12.012 . hal-02099645

\section{HAL Id: hal-02099645 \\ https://hal.science/hal-02099645}

Submitted on 15 Apr 2019

HAL is a multi-disciplinary open access archive for the deposit and dissemination of scientific research documents, whether they are published or not. The documents may come from teaching and research institutions in France or abroad, or from public or private research centers.
L'archive ouverte pluridisciplinaire HAL, est destinée au dépôt et à la diffusion de documents scientifiques de niveau recherche, publiés ou non, émanant des établissements d'enseignement et de recherche français ou étrangers, des laboratoires publics ou privés. 


\section{Author's Accepted Manuscript}

A Space-Discretized Mixed-Integer Linear Model for Air-Conflict Resolution with Speed and Heading Maneuvers

Jérémy Omer

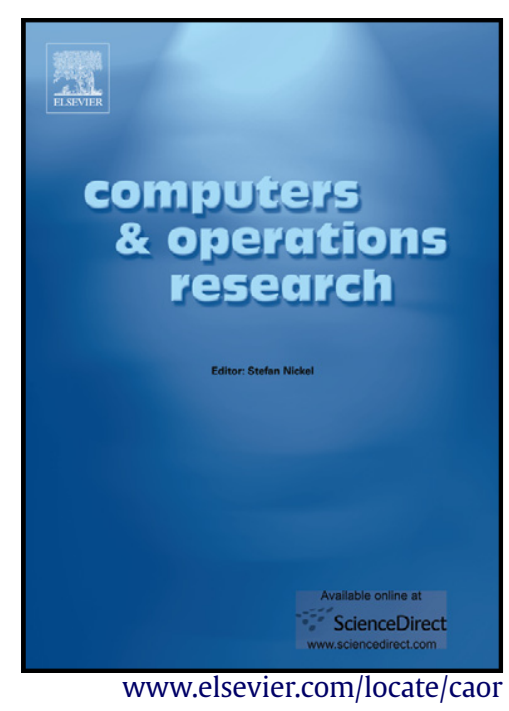

PII:

S0305-0548(15)00005-2

DOI: $\quad$ http://dx.doi.org/10.1016/j.cor.2014.12.012

Reference: CAOR3709

To appear in: Computers \& Operations Research

Received date: 4 April 2014

Revised date: 3 October 2014

Accepted date: 30 December 2014

Cite this article as: Jérémy Omer, A Space-Discretized Mixed-Integer Linear Model for Air-Conflict Resolution with Speed and Heading Maneuvers, Computers \& Operations Research, http://dx.doi.org/10.1016/j.cor.2014.12.012

This is a PDF file of an unedited manuscript that has been accepted for publication. As a service to our customers we are providing this early version of the manuscript. The manuscript will undergo copyediting, typesetting, and review of the resulting galley proof before it is published in its final citable form. Please note that during the production process errors may be discovered which could affect the content, and all legal disclaimers that apply to the journal pertain. 


\title{
A Space-Discretized Mixed-Integer Linear Model for Air-Conflict Resolution with Speed and Heading Maneuvers
}

\author{
Jérémy Omer ${ }^{\mathrm{a}, \mathrm{b}, *}$ \\ ${ }^{a}$ École Polytechnique de Montréal, 2900 bd. Edouard-Montpetit, Montreal (QC), Canada H3T 1J4 \\ ${ }^{b}$ Group for Research in Decision Analysis, HEC Montréal, 3000 ch. de la Côte-Sainte-Catherine, Montreal (QC), Canada H3T 2A7
}

\begin{abstract}
Air-conflict resolution is a bottleneck of air traffic management that will soon require powerful decision-aid systems to avoid the proliferation of delays. Since reactivity is critical for this application, we develop a mixed-integer linear model based on space discretization so that complex situations can be solved in near real-time. The discretization allows us to model the problem with finite and potentially small sets of variables and constraints by focusing on important points of the planned trajectories, including the points where trajectories intersect. A major goal of this work is to use space discretization while allowing velocity and heading maneuvers. Realistic trajectories are also ensured by considering speed vectors that are continuous with respect to time, and limits on the velocity, acceleration, and yaw rate. A classical indicator of economic efficiency is then optimized by minimizing a weighted sum of fuel consumption and delay. The experimental tests confirm that the model can solve complex situations within a few seconds without incurring more than a few kilograms of extra fuel consumption per aircraft.
\end{abstract}

Keywords: Conflict resolution, Space discretization, Air traffic control, Mixed-integer linear programming

\section{Introduction}

\subsection{Automating air traffic control}

Travelers would certainly hesitate before taking a flight if they thought there was a possibility that their aircraft would collide with another. It is the mission of air traffic control (ATC) operators to ensure in real-time that this fear remains unfounded. Based on reference horizontal and vertical separation distances, the event that must be avoided is a loss of separation, i.e., a simultaneous violation of both reference distances. When a loss of separation is predicted between two aircraft, they are said to be involved in a conflict. The ATC operators thus monitor the traffic, and when they detect a conflict, they design maneuvers that avoid a loss of separation. These maneuvers may involve a modification of speed, heading, or altitude, although altitude changes are rare for several reasons including the comfort of passengers, fuel consumption, and ease of monitoring.

Monitoring the traffic and designing maneuvers generates a heavy cognitive workload for the ATC operators. This would compromise safety if anticipative actions were not carried out to avoid dangerous situations. ATC is thus a critical stage in the overall air traffic management (ATM), since it constrains the airspace structure and traffic flow. Assuming that the forecast published by Eurocontrol [25] is correct, air traffic will undergo a 50\% increase by 2035 . In this context, severe additional regulations will be needed to deliver reasonable aircraft flows to ATC. A simulationbased study by Lehouillier et al. [13] estimates that this will lead to a 34-fold growth in delay costs if the current control procedures are unchanged. It seems that automated tools for ATC will be necessary to support this important traffic increase. Since the conflict resolution problem is the most complex task of ATC operators and presents a mathematical and algorithmic challenge, it has been widely studied.

\footnotetext{
*Tel: +15143405121 \#6051

Email address: jeremy.omer@gmail.com (Jérémy Omer)
} 


\subsection{Literature review on automated conflict resolution}

The conflict resolution problem consists in finding minimum-cost trajectories connecting two given positions with the linking constraints that a minimum separation should be ensured at all times. The result is a difficult continuoustime problem in which multiple trajectories must be simultaneously determined with additional nonconvex separation constraints. As a consequence, the existing studies all represent a compromise between the generality of the hypotheses, the realism of the model, and computational efficiency.

The most realistic models are developed in the theoretical framework of optimal control [5, 20]. The continuoustime nature of the problem is conserved, but analytical solutions can be found only for simple cases with two aircraft and a constant velocity. Based on the shapes of these analytical solutions, Bicchi and Pallottino [5] handle situations involving more aircraft and conflicts with a heuristic approach. The algorithm minimizes the total flown distance, but limits the exploration to constant speed trajectories combining circular arcs and straight lines. Raghunathan et al. [20] sample the time interval to develop a nonlinear program (NLP) that may be solved numerically. However, Borrelli et al. [6] observed rather disappointing computational results when solving the NLP, highlighting the importance of the starting point.

Several alternatives have been proposed for a rapid solution. Frese and Beyerer [11] limit the possible maneuvers to a finite set of instantaneous heading changes with constant speed or instantaneous speed changes with constant heading to solve the problem through a tree exploration. Focusing also on a finite set of maneuvers involving heading changes with constant speed, several authors have applied metaheuristic methods. For instance, Durand and Alliot [9] implement an ant colony algorithm, and Alonso-Ayuso et al. [3] develop a variable neighborhood search.

The most widespread family of algorithms is based on mixed-integer linear programs (MILPs). Moving from a complete continuous-time formulation to an MILP requires a discretization of the optimal control problem so that it can be modeled with a finite number of constraints and variables. The most obvious discretization is that used to obtain an NLP in [20]: the time interval is sampled into a finite set of time steps, and decisions are taken at each step. The separation constraints are then verified by measuring the distance between each pair of aircraft at each time step. An MILP with time discretization is for instance developed by Schouwenaars [23], by Richards and How [22] and by Omer and Farges [17]. Another strategy is to resolve the conflicts with at most one maneuver per aircraft, executing all the maneuvers simultaneously at the initial time. This may be interpreted as a particular case of time discretization in which only one time step is considered; see [19, 26].

Since the entire space may not be relevant for the conflict resolution, another discretization method, henceforth referred to as space discretization, focuses on the most interesting points of the airspace, namely those where trajectories intersect. In contrast to time discretization, the natural decision variables then represent the instants when the sampling points are flown over. Separation is then characterized by the gap between fly-over times at conflict points. This technique is used to develop models that are restricted to speed maneuvers in [27, 2, 21].

This classification of discretization techniques may also be extended to several nonlinear models. For instance, a time discretization is used in [4] and a space discretization is used in [7] to solve conflicts with speed changes only.

\subsection{Critical analysis and contribution statement}

The strength of MILPs is that algorithms can guarantee to find optimal solutions, and efficient implementations of these algorithms are fast even for large numbers of variables and constraints. For this particular application, optimality may seem insignificant since reasonable maneuvers impact only small portions of the complete trajectories. Good conflict-free trajectories, such as those determined by controllers, should not cost much more than the optimal ones. However, MILP approaches guarantee that if a feasible solution exists it will be found, and it will provide an efficient way to resolve the conflicts as long as the objective function accurately reflects real costs.

Of the modeling techniques that have been used to formulate the problem with linear constraints and objective function, time discretization appears to be classical. It enables several authors to include both speed and heading maneuvers while taking realistic constraints into account [20, 6, 10, 17]. The disadvantage of these models is that they have to sample the time horizon with a sufficiently large number of time steps to remain precise. This leads to an equally large number of variables and constraints and a potentially large computational time. In contrast, spatial discretization focuses on a small number of interesting points, so the problem is expected to be solved quickly. However, the sampling points directly depend on the predicted trajectories, which makes it hard to represent the geographical deviations that would result from heading maneuvers. For this reason, models based on a spatial discretization allow 
only speed maneuvers [21,7] or also include instantaneous altitude changes reflecting a flight-level assignment rather than dynamic altitude maneuvers [27,2]. The drawback of this assumption is that aircraft cannot dramatically modify their speed at will for reasons such as aircraft performance, passenger comfort, fuel consumption, or delay adjustments. Altitude maneuvers are usually performed to separate aircraft with a vertical motion or as last-resort safety measures ${ }^{1}$ In the context of this article, the heading maneuvers correspond better to the preferences of controllers and pilots. Moreover, since the existing models implicitly assume small speed changes that comfortably anticipate the conflicts, they leave aside several realistic features that appear in some time-discretized formulations. For instance, these space-discretized models minimize the remaining losses of separation or the amplitudes of speed changes although airlines are mostly interested in fuel consumption and delays. They also include instantaneous speed changes although acceleration should be limited.

Our main contribution is to develop a space-discretized model that allows both speed and heading maneuvers. We attempt to make the designed trajectories more realistic and to comply with operational needs arising from the traffic flow management or from the airlines. As a consequence, this study considers that

- speed vectors are continuous functions of time;

- acceleration vectors are bounded to respect the comfort of passengers;

- maneuvers should minimize the total fuel consumption;

- aircraft should revert to their planned trajectories and minimize delay.

The complexity of a situation depends on the number of aircraft it involves, on the number and on the interdependency of the conflicts, and on the geometric structure. To evaluate the model, we generate a large benchmark of artificial instances involving up to 12 aircraft engaged in 36 simultaneous potential conflicts. A recently developed timediscretized model is used as a reference for experimental comparisons. The experiments aim to provide a proof of concept for the space-discretized model and to assess its ability to find efficient and realistic trajectories in a few seconds while ensuring separation for complex situations.

Our approach is based on the problem definition and on the principles of space discretization presented in Section 2 To formulate the problem with linear constraints, the maneuvers need to be restricted to particular patterns that are described and studied in Section 3. The overall model resulting from these assumptions is then developed in Section 4. It is evaluated and analyzed through experimental tests on a large number of data sets in Section 5 .

\section{Discretizing the problem spatially}

\subsection{Problem definition}

The conflict resolution problem aims to keep a set of aircraft $\mathcal{A}$ separated on a time interval [0,T]. Let $C$ be the set of pairs of aircraft; a new trajectory is planned for each aircraft in $\mathcal{A}$ so that each pair of $C$ respects the reference separation distances.

The particular problem we are focusing on deals with planar and deterministic motion. These two restrictive hypotheses are reasonable for short-term traffic control focusing on portions of the airspace away from airports, where the altitude of an aircraft is stable most of the time. In this case, a typical value of $T$ is 10 to 15 minutes, and separation needs to be achieved through heading and speed maneuvers. The motion of the aircraft being planar, only the horizontal separation is considered. The horizontal distance between each pair of conflicting aircraft must remain greater than or equal to a given separation distance $D$.

A key feature of several research projects is to base the future ATM on a four-dimensional trajectory that represents the airspace user's preferences and complies with network structure and capacity constraints; see for instance the SESAR program [24]. This contract is usually called the business trajectory (BT). Since pilots have to implement the BTs, they provide suitable trajectory predictions that may be used by ATC. At the same time, maneuver instructions issued by ATC operators should not keep a pilot from following the BT. As a consequence, spatial recovery is ensured after every loss of separation is avoided, meaning that a succession of maneuvers should lead the aircraft back to its BT. Since a strict control of velocity may be very costly and close to impossible in practice, time recovery is not required, but it is favored by a penalty on the time gap between the deconflicted trajectory and the BT at time $T$.

\footnotetext{
${ }^{1}$ See the Eurocontro webpage http://www. eurocontrol.int/acas
} 


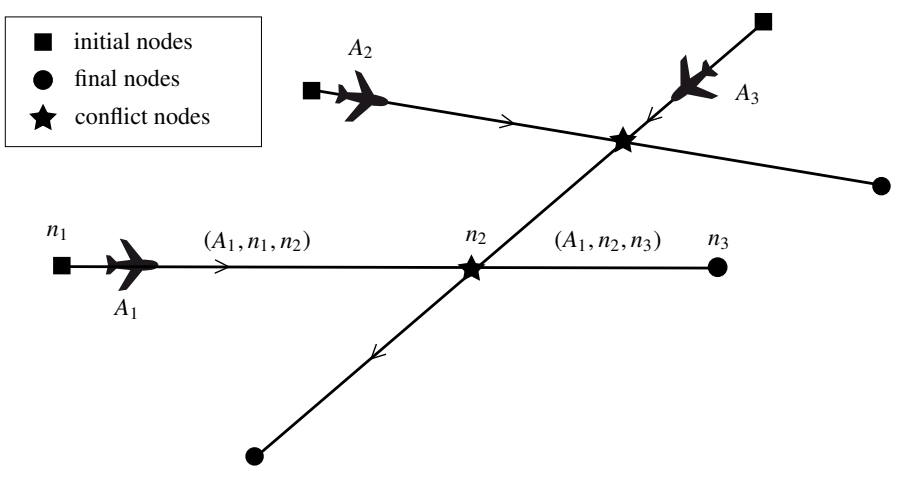

Figure 1: Nodes of a conflict graph

Finally, the trajectories are computed to minimize the total fuel consumption of the aircraft while maximizing the time recovery. Combining these two criteria provides a good measure of economic efficiency.

\subsection{Principle of the space discretization}

Space discretization focuses on the points of the airspace that are most likely to be important for the conflict resolution. These points, referred to as conflict points, are the points where the trajectories of two conflicting aircraft intersect. Although it is not explicitly identified as such, a space discretization is used in [27, 2, 7] to derive a model involving only speed maneuvers. The new difficulty that is tackled here is the inclusion of heading maneuvers.

For given traffic, the space discretization leads to a directed graph $(\mathcal{N}, \mathcal{E})$, where $\mathcal{N}$ is the union of the three following sets of nodes. The initial and final nodes are located at the initial and final positions in the BT. Conflict nodes correspond to the conflict points. Each pair of successive nodes $n_{k}$ and $n_{p}$ through which an aircraft $A_{i}$ is planned to fly defines an edge $\left(A_{i}, n_{k}, n_{p}\right) \in \mathcal{E}$. Figure 1 displays the conflict graph for a situation involving three aircraft. For clarity, only three nodes and two arcs are explicitly identified, but the graph actually contains eight nodes and seven edges.

The velocity of an aircraft on the BT reflects the preferences of the airline. Since the altitude of each flight is constant and the time horizon is small, it is logical to assume that this velocity remains constant on the considered portion of the BT. This value is henceforth referred to as the nominal velocity and denoted $V_{i}^{\text {nom }}, A_{i} \in \mathcal{A}$. The portion of the BT of $A_{i}$ corresponding to the edge $\left(A_{i}, n_{k}, n_{p}\right) \in \mathcal{E}$ is thus completely described by $\hat{O}^{k}$ and $\hat{O}^{p}$, the positions of the nodes $n_{k}$ and $n_{p}$. We can then deduce the distance $\hat{D}_{i}^{k p}$ traveled by $A_{i}$ between $\hat{O}^{k}$ and $\hat{O}^{p}$, and the times $\hat{T}_{i}^{k}$ and $\hat{T}_{i}^{p}$ when $A_{i}$ is planned to fly over $\hat{O}^{k}$ and $\hat{O}^{p}$.

The structure of the conflict graph depends only on the BTs. It thus remains unchanged during the conflict resolution. However, the maneuvers modify the characteristics of the edges.

\section{Building adapted maneuvers}

\subsection{Aircraft dynamics}

The motion being planar, the speed vector of an aircraft is described by the velocity (or speed) $V$ and the heading $\chi$; these respectively correspond to the norm and direction of the vector.

The dynamics of an aircraft are constrained by its performance and the comfort of its passengers. The performance imposes a minimum and a maximum speed, but, as specified in [18], large velocity decreases are not usually appreciated by pilots. The minimum velocity is thus determined by the performance or the pilots' preferences, whichever is the more restrictive. For $A_{i} \in \mathcal{A}$, these two extreme values are denoted $V_{i}^{\min }$ and $V_{i}^{\max }$. In addition, the comfort of the passengers requires that the velocity and heading do not change too abruptly. This leads to maximum values for their derivatives with respect to time, which are called the acceleration and yaw rate in what follows. The maximum acceleration and yaw rates are denoted $U_{i}^{\max }$ and $\omega_{i}^{\max }, A_{i} \in \mathcal{A}$. 
A reasonable level of realism is imposed on the shape of each trajectory by assuming that acceleration is stepwise constant. In other words, maneuvers are executed with a constant acceleration and yaw rate, and the speed vector remains constant between two consecutive maneuvers. This assumption is consistent with the continuity of speed with respect to time and with the general need to execute maneuvers smoothly.

\subsection{Description of the maneuvers}

In order to build a linear model, we restrict the maneuvers to any combination of the two trapezoidal patterns represented in Figure 2, and they cannot extend over more than one edge. The constraint on the heading maneuvers (see Figure 2(a) is that the aircraft has the same heading at the beginning and end of the maneuver: the purpose of the maneuver is to move the aircraft away laterally from its BT or to spatially recover it. Speed maneuvers (see Figure 2(b) start and end at the same speed: their purpose is to move the aircraft back or forward temporally (or longitudinally) from its BT.

Remark. The maneuvers are qualified as trapezoidal in reference to the shape of their graphs when heading and velocity are represented as functions of time. Indeed, if displayed as a function of time, heading would have the same global shape as velocity in Figure 2(b).

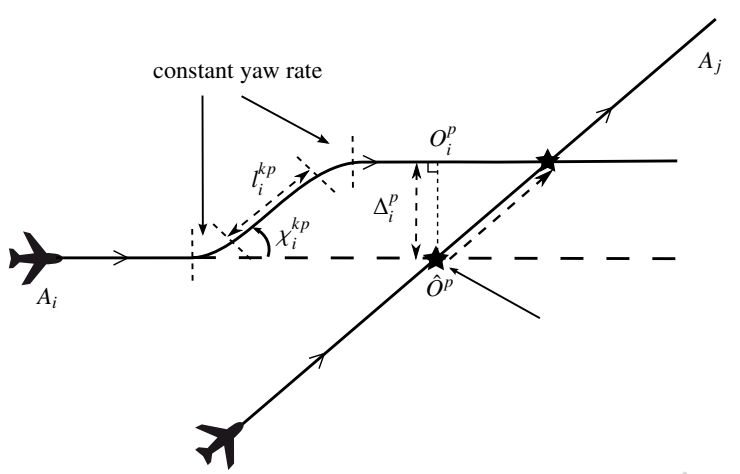

(a) Trapezoidal heading change

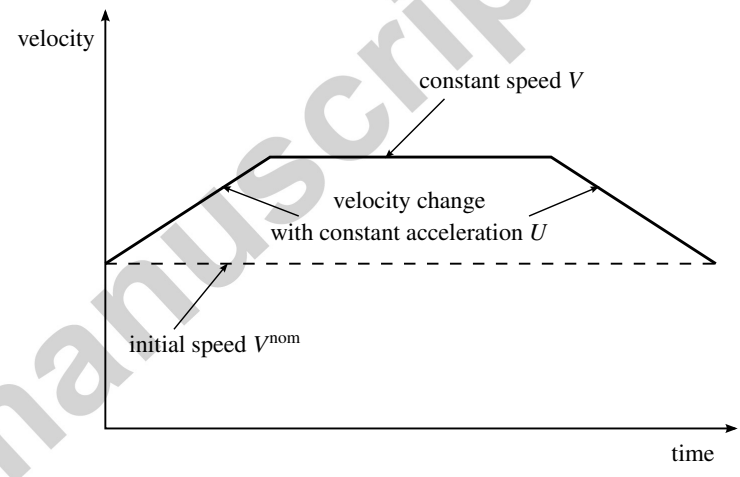

(b) Trapezoidal velocity change

Figure 2: Representation of the two permitted types of maneuvers

The maneuvers performed by an aircraft $A_{i}$ on the edge $\left(A_{i}, n_{k}, n_{p}\right)$ are described by four decision variables $\chi_{i}^{k p}$, $l_{i}^{k p}, t_{i}^{k p}$, and $d_{i}^{k p}$. Heading maneuvers are determined by $\chi_{i}^{k p}$, which is the heading change, and $l_{i}^{k p}$, the length of the segment over which the new heading is followed before the initial heading is recovered. Speed maneuvers are indirectly determined by $t_{i}^{k p}$, the temporal shift with respect to the BT created by the speed change, and $d_{i}^{k p}$, the total distance flown during the maneuver. Clearly, a maneuver creates an offset with respect to the BT, so a new set of parameters is needed to characterize the resulting trajectory in the conflict graph. One simple approach is an orthogonal projection of the important points of the BT onto the modified trajectory (see Figure 2(a)), in order to describe the latter relative to the BT. For the edge $\left(A_{i}, n_{k}, n_{p}\right)$, we thus define:

- $O_{i}^{k}, O_{i}^{p}$ : orthogonal projections of $\hat{O}^{k}$ and $\hat{O}^{p}$ onto the modified trajectory of $A_{i}$;

- $\Delta_{i}^{k}, \Delta_{i}^{p}$ : directed distances from $\hat{O}^{k}$ to $O_{i}^{k}$ and $\hat{O}^{p}$ to $O_{i}^{p}$, whose signs depend on an arbitrary orientation of space; they may also be referred to as lateral shifts at $n_{k}$ and $n_{p}$;

- $T_{i}^{k}, T_{i}^{p}$ : times when $A_{i}$ flies over $O_{i}^{k}$ and $O_{i}^{p}$.

- $D_{i}^{k p}$ : distance traveled by $A_{i}$ between $O_{i}^{k}$ and $O_{i}^{p}$.

Remark. Notice that position $O_{i}^{k}$ depends on the aircraft while $\hat{O}^{k}$ depends only on the node. This means that several different characteristic positions may refer to a single node, but not to several aircraft. In particular, this is the case at a conflict node if one of the aircraft has previously performed a heading maneuver. 


\subsection{Study of the maneuvers}

\subsubsection{Velocity changes}

Clearly, the temporal shift created by a velocity change is not a flight parameter that can easily be communicated to a pilot. The following proposition relates the value of this shift to the flight parameters.

Proposition 1. Consider a trapezoidal speed maneuver in which the velocity changes from $V^{\text {nom }}$ to $V$. Let $U$ be the absolute value of the constant acceleration during the two speed changes and $d$ be the total distance flown during the maneuver. The value of the time shift created by the maneuver, i.e., the difference between the duration of the maneuver and the time needed to fly $d$ in the BT, is:

$$
t=2 \frac{\left|V^{\mathrm{nom}}-V\right|}{U}+\frac{1}{V}\left(d-\frac{\left|\left(V^{\mathrm{nom}}\right)^{2}-V^{2}\right|}{U}\right)-\frac{d}{V^{\mathrm{nom}}} .
$$

Proof. Each acceleration phase lasts $\left|V^{\text {nom }}-V\right| / U$. By integrating acceleration twice, we see that the distance flown during the two phases is equal to $\left|\left(V^{\text {nom }}\right)^{2}-V^{2}\right| / U$. As a result, the total time that is necessary to achieve the entire maneuver is

$$
T=2 \frac{\left|V^{\mathrm{nom}}-V\right|}{U}+\frac{1}{V}\left(d-\frac{\left|\left(V^{\mathrm{nom}}\right)^{2}-V^{2}\right|}{U}\right) .
$$

Since the BT is flown with a constant speed $V^{\text {nom }}$, the temporal shift is thus given by $T-d / V^{\text {nom }}$, which leads to the expression of $t$ in (1).

Proposition 1 allows us to derive bounds ensuring that valid values of speed and acceleration always match the chosen temporal shift. If we insert the bounds on acceleration and velocity into (1), it indeed appears that the extremal values of the temporal shift are obtained when acceleration is maximal and velocity is either minimal or maximal. For an edge $\left(A_{i}, n_{k}, n_{p}\right)$, the temporal shift created by a trapezoidal speed maneuver is thus constrained by

$$
\frac{d_{i}^{k p}}{V_{i}^{\max }}-\frac{d_{i}^{k p}}{V_{i}^{\text {nom }}}+\frac{\left(V_{i}^{\text {nom }}-V_{i}^{\max }\right)^{2}}{U_{i}^{\max } V_{i}^{\max }} \leq t_{i}^{k p} \leq \frac{d_{i}^{k p}}{V_{i}^{\min }}-\frac{d_{i}^{k p}}{V_{i}^{\text {nom }}}-\frac{\left(V_{i}^{\text {nom }}-V_{i}^{\min }\right)^{2}}{U_{i}^{\max } V_{i}^{\min }} .
$$

If no heading maneuver is performed, an upper bound on $d_{i}^{k p}$ is $\hat{D}_{i}^{k p}$, the length of the edge in the BT. The general case is studied in Section 4

Remark. The actual flight parameters $V$ and $U$ do not have to appear in the model as long as (2) ensures that valid values exist. They are computed in a post-resolution process that can take additional constraints into account if necessary.

\subsubsection{Heading changes}

Given an edge $\left(A_{i}, n_{k}, n_{p}\right)$ and a heading maneuver characterized by $l_{i}^{k p}$ and $\chi_{i}^{k p}$, we now derive linear relations that specify the effect of the maneuver on the characteristics of the trajectory. In particular, assuming $T_{i}^{k}$ and $\Delta_{i}^{k}$ are known, it is necessary to determine $D_{i}^{k p}, T_{i}^{p}$, and $\Delta_{i}^{p}$.

As mentioned above, the velocity is not a variable of the model, so the velocity of the aircraft while turning is approximated by the nominal velocity. This value is chosen because it is usually the center of the interval of valid values. Since the size of the velocity interval is small when compared to the nominal value, this approximation is reasonable if the turn does not last too long. For this reason, the aircraft turns with the maximum yaw rate $\omega^{\text {max }}$.

Figure 3 shows a constant turn with yaw rate $\omega^{\text {max }}$. Referring to this figure, we may compute the actual distance flown between $O_{i}^{k}$ and $O_{i}^{p}$, and the lateral shift at $n_{p}$ :

$$
\begin{aligned}
D_{i}^{k p} & =\hat{D}_{i}^{k p}+2 \frac{V_{i}^{\mathrm{nom}}}{\omega^{\max }}\left(\left|\chi_{i}^{k p}\right|-\sin \left|\chi_{i}^{k p}\right|\right)+l_{i}^{k p}\left(1-\cos \chi_{i}^{k p}\right), \\
\Delta_{i}^{p} & =\Delta_{i}^{k}+2 \operatorname{sign}\left(\chi_{i}^{k p}\right) \frac{V_{i}^{\mathrm{nom}}}{\omega^{\max }}\left(1-\cos \chi_{i}^{k p}\right)+l_{i}^{k p} \sin \chi_{i}^{k p} .
\end{aligned}
$$




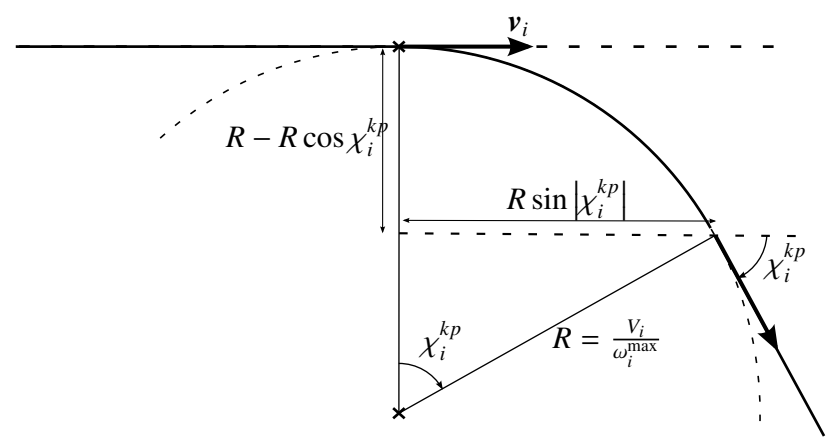

Figure 3: Geometric representation of a constant turn

The above expressions are clearly nonlinear since they contain trigonometric expressions depending on the decision variables and products involving both $l_{i}^{k p}$ and $\chi_{i}^{k p}$. They can however be made linear by restricting the possible values of heading changes to a finite set of $N_{\chi}$ values, denoted $\left\{\chi_{n}\right\}_{n=1, \ldots, N_{\chi}}$. With this in mind, we introduce two sets of decision variables: $\left\{\epsilon_{i, n}^{k p}\right\}_{n=1, \ldots, N_{\chi}}$ and $\left\{l_{i, n}^{k p}\right\}_{n=1, \ldots, N_{\chi}}$. The first variables are binary and model the choice of the heading value, meaning that $\chi_{i}^{k p}=\chi_{n}$ if and only if $\epsilon_{i, n}^{k p}=1$. The second set of variables can be interpreted as a projection of $l_{i}^{k p}$ onto the set of possible choices. More formally, $l_{i, n}^{k p}=\epsilon_{i, n}^{k p} l_{i}^{k p}, \forall n \in\left\{1, \ldots, N_{\chi}\right\}$. These properties are ensured by introducing the following linear constraints into the model for each edge $\left(A_{i}, n_{k}, n_{p}\right)$ :

$$
\begin{aligned}
& \chi_{i}^{k p}=\sum_{n=1}^{N_{\chi}} \epsilon_{i, n}^{k p} \chi_{n}, \\
& \sum_{n=1}^{N_{\chi}} \epsilon_{i, n}^{k p} \leq 1, \\
& l_{i}^{k p}=\sum_{n=1}^{N_{\chi}} l_{i, n}^{k p}, \\
& l_{i, n}^{k p} \leq \frac{\hat{D}_{i}^{k p}}{\cos \chi_{n}} \epsilon_{i, n}^{k p}, \quad \forall n \in\left\{1, \ldots, N_{\chi}\right\} .
\end{aligned}
$$

Constraint (5) ensures that at most one heading is chosen on $\left(A_{i}, n_{k}, n_{p}\right)$, and (4) sets the heading to the chosen value. The bounds (7) imply that $\epsilon_{i, n}^{k p}=0 \Rightarrow l_{i, n}^{k p}=0$, but they do not limit $l_{i, n}^{k p}$ if $\epsilon_{i, n}^{k p}=1$, because $\hat{D}_{i}^{k p} / \cos \chi_{n}$ is a valid upper bound of $l_{i}^{k p}$. In theory, any valid upper bound could replace $\hat{D}_{i}^{k p} / \cos \chi_{n}$ in (7) to obtain an equivalent model. The continuous relaxation of the MILP can then be made tighter by finding another upper bound with a lower value. To improve the relaxation, we observe that a heading maneuver always starts and ends with constant turns whose projections onto the BT are $\frac{V_{i}^{\text {nom }}}{\omega^{\max }} \sin \left|\chi_{i}^{k p}\right|$ long. As a consequence, the following upper bound is valid and has a lower value:

$$
l_{i, n}^{k p} \leq\left(\frac{\hat{D}_{i}^{k p}}{\cos \chi_{n}}-2 \frac{V_{i}^{\mathrm{nom}}}{\omega^{\max }} \sin \left|\chi_{n}\right|\right) \cdot \epsilon_{i, n}^{k p} .
$$

If we insert $\left\{\epsilon_{i, n}^{k p}\right\}_{n=1, \ldots, N_{\chi}}$ and $\left\{l_{i, n}^{k p}\right\}_{n=1, \ldots, N_{\chi}}$ into $[3$, the edge's length and the lateral shift become linear functions of 
the decision variables:

$$
\begin{array}{r}
D_{i}^{k p}=\hat{D}_{i}^{k p}+\sum_{n=1}^{N_{\chi}}\left[2 \epsilon_{i, n}^{k p} \times \frac{V_{i}^{\mathrm{nom}}}{\omega^{\max }}\left(\left|\chi_{n}\right|-\left|\sin \chi_{n}\right|\right)+l_{i, n}^{k p}\left(1-\cos \chi_{n}\right)\right], \\
\Delta_{i}^{p}=\Delta_{i}^{k}+\sum_{n=1}^{N_{\chi}}\left[2 \epsilon_{i, n}^{k p} \times \operatorname{sign}\left(\chi_{n}\right) \frac{V_{i}^{\mathrm{nom}}}{\omega^{\max }}\left(1-\cos \chi_{n}\right)+l_{i, n}^{k p} \sin \chi_{n}\right] .
\end{array}
$$

Finally, it will take the aircraft a time $D_{i}^{k p} / V_{i}^{\text {nom }}$ to fly from $O_{i}^{k}$ to $O_{i}^{p}$ if the velocity remains constant. If a speed maneuver is performed, the additional time shift is equal to $t_{i}^{k p}$. As a consequence, the fly-over time at $n_{p}$ is obtained by

$$
T_{i}^{p}=T_{i}^{k}+t_{i}^{k p}+\frac{D_{i}^{k p}}{V_{i}^{\text {nom }}} .
$$

Remark. Altitude maneuvers are not included in the model, because they would make it unnecessarily complex. These maneuvers are not appropriate for short-term separation, since they are uncomfortable for passengers, inefficient in terms of fuel consumption, and hard for ATC operators to monitor. However, flight-level assignments could be included as in [27, 2]. They would make it necessary to simultaneously consider several flight levels, but no additional difficulty would arise since they do not model the dynamics of the aircraft. As mentioned in the Introduction, these assignments would be more relevant for mid-term trajectory planning.

\section{Modeling the complete problem}

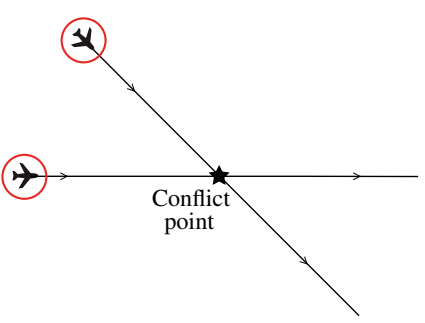

(a) Crossing conflict $\left(\in C_{C}\right)$

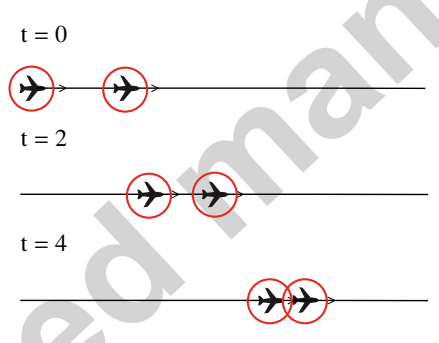

(b) Trailing conflict $\left(\in C_{T}\right)$

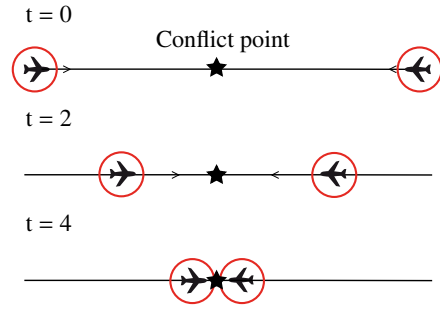

(c) Frontal conflict $\left(\in C_{F}\right)$

Figure 4: Three categories of conflicts

\subsection{Separation constraints}

Consider $\left(A_{i}, A_{j}\right) \in C$, and let $\theta_{i j}$ be the directed angle between the speed vectors of $A_{i}$ and $A_{j}$. Let $n_{k}$ be the associated conflict node.

In contrast to time discretization, space discretization does not allow us to treat all the conflicts with the same set of constraints. In particular, if $A_{i}$ and $A_{j}$ fly on the same route, in the same or opposite directions, the usual constraints that are found in the models involving only speed maneuvers do not apply. In this section, we develop a set of constraints for each category of conflicts represented in Figure 4 The sets of crossing, trailing, and frontal conflicts are respectively $C_{C}, C_{T}$, and $C_{F}$. For practical reasons, the node $n_{k}$ where a conflict between $A_{i}$ and $A_{j}$ occurs is added to the pair $\left(A_{i}, A_{j}\right)$, so the conflict is now denoted $\left(A_{i}, A_{j}, n_{k}\right)$.

\subsubsection{Crossing conflicts}

While separation constraints focus on the positions of conflicting aircraft in a time-discretized model, for spatial discretization they focus on fly-over times. To be specific, if $\left(A_{i}, A_{j}, n_{k}\right) \in C_{C}$, the instants $A_{i}$ and $A_{j}$ fly over the 


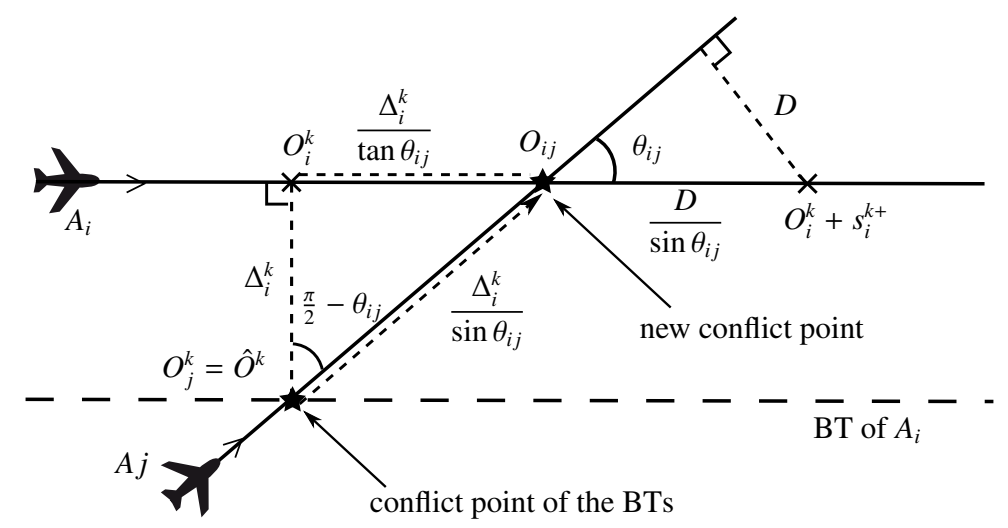

Figure 5: Effect of a lateral shift on a crossing conflict

conflict point must be separated by a minimum time $T_{i j}^{\min }$, depending on their speeds and the angle between their BTs. If the speed vectors of both aircraft are constant, and the trajectories intersect with a directed angle equal to $\theta_{i j}, T_{i j}^{\mathrm{min}}$ may be computed as in [27]:

$$
T_{i j}^{\mathrm{min}}=\frac{D}{V_{i}^{\mathrm{nom}} V_{j}^{\mathrm{nom}}\left|\sin \theta_{i j}\right|} \sqrt{\left(V_{i}^{\mathrm{nom}}\right)^{2}+\left(V_{j}^{\mathrm{nom}}\right)^{2}-2 V_{i}^{\mathrm{nom}} V_{j}^{\mathrm{nom}} \cos \theta_{i j}}
$$

where $D$ is the reference separation distance. Separation may be achieved with $A_{i}$ passing either in front of or behind $A_{j}$. If only speed changes are performed, the conflict occurs at $\hat{O}^{k}$, and this disjunctive constraint has a linear expression involving a binary variable $\delta_{i j}$ and a large constant value $M$ :

$$
T_{i}^{k}-T_{j}^{k} \geq T_{i j}^{\min }-M \delta_{i j}, \quad \text { and } \quad T_{j}^{k}-T_{i}^{k} \geq T_{i j}^{\min }-M\left(1-\delta_{i j}\right) .
$$

For instance, if the decision is that $A_{i}$ passes first, $\delta_{i j}$ is set to 0 and the two constraints become $T_{i}^{k}-T_{j}^{k} \geq T_{i j}^{\mathrm{min}}$ and $T_{j}^{k}-T_{i}^{k} \geq T_{i j}^{\min }-M$. The first constraint then ensures the horizontal separation, and the second is always satisfied if $M$ is large enough. This technique is common when modeling disjunctive constraints with linear expressions; it is usually referred to as the big-M method.

Figure 5 shows a section of Figure 2(a) to illustrate the effect of a lateral shift on a crossing conflict. For simplicity only the shift of $A_{i}$ is pictured, but similar effects would arise if $A_{j}$ was also shifted. The figure shows that a lateral shift of $A_{i}$ translates the conflict point from its initial position $\hat{O}^{k}$ to $O_{i j}$. Since the fly-over times of $A_{i}$ and $A_{j}$ at $n_{k}$ are measured with respect to $O_{i}^{k}$ and $O_{j}^{k}$, the times over $O_{i j}$ are not directly given by $T_{i}^{k}$ and $T_{i}^{p}$. The times over the conflict point are important for the separation, so (12) must be modified accordingly to take lateral shifts into account. Proposition 2 specifies the resulting separation constraints. In the statement of the proposition, the subscripts on $M_{i j}$ highlight that the big-M value may depend on the pair $\left(A_{i}, A_{j}\right)$. Also, $\left|O O^{\prime}\right|$ is the directed distance between $O$ and $O^{\prime}$ along the trajectory of $A_{i} \in \mathcal{A}$ for any two points $O$ and $O^{\prime}$ of this trajectory.

Proposition 2. Let $\left(A_{i}, A_{j}, n_{k}\right) \in C_{C}$, and assume that the speed vectors of $A_{i}$ and $A_{j}$ are constant and equal to those of their BTs. Then separation is guaranteed if and only if

$$
T_{i}^{k}+\frac{\left|O_{i}^{k} O_{i j}\right|}{V_{i}^{\text {nom }}}-T_{j}^{k}-\frac{\left|O_{j}^{k} O_{i j}\right|}{V_{j}^{\text {nom }}} \geq T_{i j}^{\min }-M_{i j} \cdot \delta_{i j}, \text { and } T_{j}^{k}+\frac{\left|O_{j}^{k} O_{i j}\right|}{V_{j}^{\text {nom }}}-T_{i}^{k}-\frac{\left|O_{i}^{k} O_{i j}\right|}{V_{i}^{\text {nom }}} \geq T_{i j}^{\min }-M_{i j} \cdot\left(1-\delta_{i j}\right),
$$

As illustrated in Figure 5 , the shift $\Delta_{i}^{k}$ moves the conflict point a distance $\Delta_{i}^{k} / \tan \theta_{i j}$ along the trajectory of $A_{i}$ and a distance $\Delta_{i}^{k} / \sin \theta_{i j}$ along the trajectory of $A_{j}$. The same considerations with respect to a shift of $A_{j}$ leads to the 
following expressions for the directed distances:

$$
\left|O_{i}^{k} O_{i j}\right|=\frac{\Delta_{i}^{k}}{\tan \theta_{i j}}-\frac{\Delta_{j}^{k}}{\sin \theta_{i j}} \text { and }\left|O_{j}^{k} O_{i j}\right|=-\frac{\Delta_{j}^{k}}{\tan \theta_{i j}}+\frac{\Delta_{i}^{k}}{\sin \theta_{i j}} .
$$

This emphasizes the fact that the constraints in (13) are linear.

To get the tightest possible constraints, the big-M values in (13) should be chosen by finding a lower bound of the left-hand sides. For instance, if $l b$ is such a lower bound for the first constraint then, for any $M_{i j} \geq T_{i j}^{\min }-l b, \delta_{i j}=1$ implies that the constraint is satisfied whatever the values of the decision variables. Clearly, the most interesting value is $T_{i j}^{\min }-l b$. Assuming that $\hat{O}^{i}$ and $\hat{O}^{j}$ are the initial positions of $A_{i}$ and $A_{j}$, and denoting $\chi^{\max }$ the maximum allowed heading change, then the lateral shifts at $n_{k}$ are bounded by

$$
\left|\Delta_{i}^{k}\right| \leq\left|\hat{O}^{i} \hat{O}^{k}\right| \cdot \tan \chi^{\max }=\Delta_{i k}^{\max }, \text { and }\left|\Delta_{j}^{k}\right| \leq\left|\hat{O}^{j} \hat{O}^{k}\right| \cdot \tan \chi^{\max }=\Delta_{j k}^{\max }
$$

Relying on equations (13) and (14), a relatively tight constraint is then obtained by setting

$$
M_{i j}=T_{i j}^{\mathrm{min}}-T_{i}^{k}+T_{j}^{k}+\Delta_{i k}^{\mathrm{max}} \cdot\left|\frac{1}{V_{i}^{\mathrm{nom}} \tan \theta_{i j}}-\frac{1}{V_{j}^{\mathrm{nom}} \sin \theta_{i j}}\right|+\Delta_{j k}^{\mathrm{max}} \cdot\left|\frac{1}{V_{j}^{\mathrm{nom}} \tan \theta_{i j}}-\frac{1}{V_{i}^{\mathrm{nom}} \sin \theta_{i j}}\right|
$$

The separation constraints in (13) are valid only if the speed vectors are constant. Obviously, speed vectors cannot remain constant over the whole time horizon if maneuvers need to be performed. The following proposition allows us to specify the segment where the speed vectors are constant.

Proposition 3. Let $s_{i}$ and $s_{j}$ be the curvilinear abscissas of $A_{i}$ and $A_{j}$ with respective origins at $O_{i}^{k}$ and $O_{j}^{k}$. The constraints in (13) guarantee the separation of the aircraft if their speed vectors are constant for

$$
\left|O_{i}^{k} O_{i j}\right|-\frac{D}{\sin \left|\theta_{i j}\right|} \leq s_{i} \leq\left|O_{i}^{k} O_{i j}\right|+\frac{D}{\sin \left|\theta_{i j}\right|} \text { and }\left|O_{j}^{k} O_{i j}\right|-\frac{D}{\sin \left|\theta_{i j}\right|} \leq s_{j} \leq\left|O_{j}^{k} O_{i j}\right|+\frac{D}{\sin \left|\theta_{i j}\right|} .
$$

Proof. The result is obtained by noticing that outside these intervals, the distance between the courses of the two trajectories is larger than $D$, as depicted in Figure 5.

If several conflicts involve a given $A_{i} \in \mathcal{A}$ at node $n_{k}$, the segment where the speed vector is constant is the union of the intervals imposed by each conflict. The two extremities of this segment are given by the variables $s_{i}^{k-} \leq 0$ and $s_{i}^{k+} \geq 0$, whose signs impose that the speed vector is always constant at $O_{i}^{k}$. The concrete effect of having the speed vector constant on a segment centered on a given node is that less space is available on the edges upstream and downstream. This allows us to clarify the bounds on the decision variables. For an edge $\left(A_{i}, n_{k}, n_{p}\right)$, the distance available for speed changes implies that

$$
d_{i}^{k p} \leq D_{i}^{k p}+s_{i}^{p-}-s_{i}^{k+} .
$$

Additionally, the length of the orthogonal projection of a heading maneuver onto the BT must not exceed $\hat{D}_{i}^{k p}+s_{i}^{p-}-$ $s_{i}^{k+}$. When we take constant turns into account, the distance for which an aircraft may follow a new heading is bounded by

$$
l_{i, n}^{k p} \cos \chi_{n} \leq \hat{D}_{i}^{k p}+s_{i}^{p-}-s_{i}^{k+}-2 \epsilon_{i, n}^{k p} \frac{V_{i}^{\mathrm{nom}}}{\omega^{\max }}\left(\left|\sin \chi_{n}\right|\right), \forall n \in\left\{1, \ldots, N_{\chi}\right\} .
$$

\subsubsection{Trailing conflicts}

These conflicts include only the situations in which one aircraft follows another without intention or permission to overtake. Let $A_{i}$ and $A_{j}$ be two aircraft flying over the edge connecting $n_{k}$ and $n_{p}$, and assume without loss of generality that $A_{i}$ is in front of $A_{j}$.

Proposition 4. Assume that the norms of the orthogonal projections of the speed vectors of $A_{i}$ and $A_{j}$ onto their respective BTs are constant on the edge connecting $n_{k}$ and $n_{p}$. Let $\tilde{V}_{i}^{k p}$ and $\tilde{V}_{j}^{k p}$ be these velocities, and $\chi_{i}^{\max }$ be the 
largest heading change allowed for $A_{i}$. The separation is guaranteed on this edge if

$$
T_{i}^{k}+\frac{D}{V_{i}^{\min } \cos \chi_{i}^{\max }} \leq T_{j}^{k} \quad \text { and } \quad T_{j}^{p}-\frac{D}{V_{j}^{\max }} \geq T_{i}^{p} .
$$

Proof. Because of the bounds on the velocity, $\tilde{V}_{i}^{k p} \geq V_{i}^{\min } \cos \chi_{i}^{\max }$ and $\tilde{V}_{j}^{k p} \leq V_{j}^{\max }$. The constraints in (20) then ensure that the projected positions of $A_{i}$ and $A_{j}$ are separated by a distance $D$ at each extremity of the edge. The projected velocities being constant, the separation between the projected positions is also guaranteed on the whole edge, which is sufficient to ensure separation between the actual positions of the aircraft.

The assumption that the projected velocities are constant does not hold if one of the aircraft performs a maneuver on this edge. Let $s_{i}^{\perp}(t)$ be the curvilinear abscissa of the position of $A_{i}$ projected onto its BT, and $\tilde{s}_{i}(t)$ be the curvilinear abscissa of a point moving on the BT with a constant velocity equal to $\tilde{V}_{j}^{k p}$, the average value of the norm of the projection of the speed vector of $A_{i}$ onto its BT. If we take $O_{i}^{k}$ as the origin of the abscissas, proposition 4 indicates that $\tilde{s}_{i}(t)$ and $\tilde{s}_{j}(t)$ are at least separated by a distance $D$ if the constraints $(20)$ are satisfied. This is not sufficient for the separation of $A_{i}$ and $A_{j}$, but the same result with $s_{i}^{\perp}(t)$ and $s_{j}^{\perp}(t)$ would be. The values of $s_{i}^{\perp}(t)$ and $s_{j}^{\perp}(t)$ cannot be known without a complete description of the maneuvers of $A_{i}$ and $A_{j}$, which are computed only after the model has been solved. This is actually an advantage in this case, because the start of each maneuver can then be chosen to ensure that separation is guaranteed. For instance, if a heading maneuver is to be performed, ending the maneuver at the end of the edge implies that $s_{i}^{\perp}(t) \leq \tilde{s}_{i}(t), \forall T_{i}^{k} \geq t \leq T_{i}^{p}$. Conversely, with similar notation for $A_{j}$, starting a heading maneuver at the beginning of the edge implies that $s_{j}^{\perp}(t) \geq \tilde{s}_{j}(t), \forall T_{j}^{k} \leq t \leq T_{j}^{p}$. Although additional calculations are necessary for a full proof, this discussion shows that the starting times of the maneuvers can be set so that constraints (20) guarantee the separation for trailing conflicts.

Since the end of one edge is the beginning of the next, the constraints in (20) are summarized by a single constraint for each $\left(A_{i}, A_{j}, n_{k}\right) \in C_{T}$ :

$$
T_{i}^{k} \leq T_{j}^{k}-\frac{D}{\min \left(V_{i}^{\min } \cos \chi_{i}^{\max }, V_{j}^{\max }\right)} .
$$

\subsubsection{Frontal conflicts}

Clearly, when two aircraft are in frontal conflict, the two trajectories do not intersect at a unique point of space. As a consequence, the conflict point is defined differently than for the crossing conflicts. It is the point where the projections of the trajectories onto the BTs collide (see Figure 4(c)).

Let $\left(A_{i}, A_{j}, n_{k}\right) \in C_{F}$. The separation must be performed spatially by ensuring that the lateral shifts satisfy $\left|\Delta_{i}^{k}-\Delta_{j}^{k}\right| \geq D$, and that this separation remains stable in the vicinity of the conflict. The absolute value reflects the two possible configurations $\left(\Delta_{i}^{k}>\Delta_{j}^{k}\right.$ or $\left.\Delta_{i}^{k}<\Delta_{j}^{k}\right)$, but the constraint is not linear. As for the crossing conflicts, the separation is modeled with a binary decision variable $\delta_{i j}$ and a large constant value $M_{i j}$ :

$$
\Delta_{i}^{k}-\Delta_{j}^{k} \geq D-M_{i j} \cdot \delta_{i j}, \quad \text { and } \quad \Delta_{j}^{k}-\Delta_{i}^{k} \geq D-M_{i j} \cdot\left(1-\delta_{i j}\right)
$$

It is then sufficient to maintain the speed vectors of the aircraft constant on a segment of length $D$ centered on the conflict point. As in Section 4.1.1, the bounds on the lateral shifts exhibited in equation (15) are used to compute big-M values leading to tight constraints:

$$
M_{i j}=D+\Delta_{i k}^{\max }+\Delta_{j k}^{\max }
$$

\subsection{Objective function}

\subsubsection{Fuel consumption}

As in [26], the BADA user manual [16] is used as the reference physical model from which the instantaneous fuel consumption per unit of time $C_{t}$ is derived:

$$
C_{t}(t)=c_{1}\left(1+\frac{V(t)}{c_{2}}\right)\left(\alpha V_{i}(t)^{2}+\frac{\beta}{V(t)^{2}}+m \frac{\mathrm{d} V(t)}{\mathrm{d} t}\right),
$$


where $m$ is the mass of the aircraft, $c_{1}, c_{2}$ depend on the aircraft type, and $\alpha, \beta$ depend on the aircraft type, and on the current altitude and mass of the aircraft. Since the aircraft are assumed to fly at their nominal speeds at the beginning and at the end of the time horizon, the term involving the derivative of the velocity nullifies when integrated over the time interval. Moreover, the altitude is constant and variations in mass can be neglected over a fifteen-minute interval, so $C_{t}$ is a rational fraction depending only on the velocity. The fuel consumption per unit of distance $C_{d}$ is then obtained by

$$
C_{d}(V)=\frac{C_{t}(V)}{V} \approx\left(\frac{c_{1}}{V}+\frac{c_{1}}{c_{2}}\right)\left(\alpha V+\frac{\beta}{V^{3}}\right)
$$

To estimate the fuel consumption of an aircraft $A_{i}$, we approximate its velocity by its average value on each edge. Clearly, this approximation is needed only during the speed maneuvers. For the edge $\left(A_{i}, n_{k}, n_{p}\right)$, the average value $\widetilde{V}_{i}^{k p}$ is deduced from

$$
t_{i}^{k p}=d_{i}^{k p}\left(\frac{1}{\widetilde{V}_{i}^{k p}}-\frac{1}{V_{i}^{\text {nom }}}\right) \text {, which means that } \widetilde{V}_{i}^{k p}=\left(\frac{t_{i}^{k p}}{d_{i}^{k p}}+\frac{1}{V_{i}^{\text {nom }}}\right)^{-1} .
$$

On this edge, a distance $d_{i}^{k p}$ is flown at $\widetilde{V}_{i}^{k p}$, and the rest is flown at $V_{i}^{\text {nom }}$. The fuel consumption is thus given by

$$
Z_{i}^{k p}=d_{i}^{k p} C_{d, i}\left(\widetilde{V}_{i}^{k p}\right)+\left(D_{i}^{k p}-d_{i}^{k p}\right) C_{d, i}\left(V_{i}^{\mathrm{nom}}\right)
$$

$C_{d, i}\left(\widetilde{V}_{i}^{k p}\right)$ is not a linear function of the decision variables, so a stepwise-linear approximation is used. The particular function that is approximated is $t \mapsto C_{d, i}\left(\left(t+\frac{1}{V_{i}^{\text {nom }}}\right)^{-1}\right)$. Let $N_{z}$ be the number of segments and $y=\alpha_{i}^{n} x+\beta_{i}^{n}$, $n \in\left\{1, \ldots, N_{Z}\right\}$ the corresponding equations. Although no general statement can be made by examining the general form of $C_{d}$, the stepwise-linear function happens to be convex on $\left[V^{\min }, V^{\max }\right]$ for the main types of aircraft. Therefore,

$$
C_{d, i}\left(\widetilde{V}_{i}^{k p}\right) \approx \max _{n \in\left\{1, \ldots, N_{Z}\right\}}\left\{\alpha_{i}^{n} \frac{t_{i}^{k p}}{d_{i}^{k p}}+\beta_{i}^{n}\right\}, \text { hence } Z_{i}^{k p} \approx \max _{n \in\left\{1, \ldots, N_{Z}\right\}}\left\{\alpha_{i}^{n} t_{i}^{k p}+\beta_{i}^{n} d_{i}^{k p}\right\}+\left(D_{i}^{k p}-d_{i}^{k p}\right) C_{d, i}\left(V_{i}^{\mathrm{nom}}\right) .
$$

As a result, the decision variable $z_{i}^{k p}$ approaches the fuel consumption if it is minimized and constrained by

$$
z_{i}^{k p} \geq \alpha_{i}^{n} t_{i}^{k p}+\beta_{i}^{n} d_{i}^{k p}+\left(D_{i}^{k p}-d_{i}^{k p}\right) C_{d, i}\left(V_{i}^{\mathrm{nom}}\right), \forall n \in\left\{1, \ldots, N_{Z}\right\}
$$

As an illustration, Figure 6 shows the fuel consumption function of an A320 at 33,000 feet together with the stepwise-linear approximation obtained with $N_{Z}=4$.

\subsubsection{Space and time recovery}

Assume that $n_{f}$ is the final node that $A_{i} \in \mathcal{A}$ reaches at time $T$ in the BT. Spatial recovery is guaranteed if and only if $\Delta_{i}^{f}=0$.

The final time shift with the BT is given by $\Delta_{T, i}=T_{i}^{f}-T$. This shift needs to be penalized to obtain consistent trajectories, but the derivation of the cost of time is not as straightforward as that of fuel. However, it seems natural that the delay penalty should compensate for the fuel cost that would be incurred by a complete time recovery. We emphasize that this recovery may occur after the time horizon or may even never be performed if the airline does not require it, but it remains a valid reference.

First assume that $A_{i}$ is late with regards to its BT, i.e., $\Delta_{T, i}<0$. Since no emergency motivates the recovery, it would be reasonable to execute it with a velocity

$$
V_{i}^{-}=\frac{V_{i}^{\max }+V_{i}^{\text {nom }}}{2}
$$




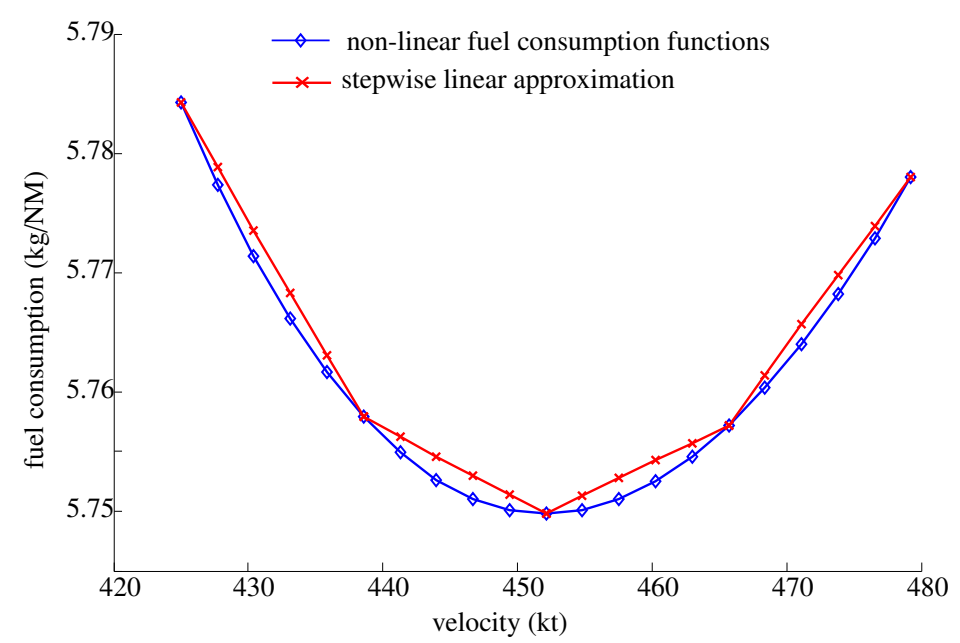

Figure 6: Fuel consumption per nautical mile for an Airbus A320 at 33,000 feet

Let $D$ be the distance flown during the time recovery. Since the BT is flown with a velocity $V_{i}^{\text {nom }}$, it is necessary that

$$
\frac{D}{V_{i}^{-}}-\frac{D}{V_{i}^{\mathrm{nom}}}=\Delta_{T, i} \Leftrightarrow D=\Delta_{T, i} \cdot \frac{V_{i}^{\mathrm{nom}}-V_{i}}{V_{i}^{-} \cdot V_{i}^{\mathrm{nom}}}
$$

Denoting $\rho_{i}^{-}$the penalty associated with a negative time shift, the delay cost compensates for the fuel consumption if

$$
C_{d, i}\left(V_{i}^{-}\right) \cdot D=\rho_{i}^{-} \cdot \Delta_{T, i} \Leftrightarrow \rho_{i}^{-}=C_{d, i}\left(V_{i}^{-}\right) \cdot D \cdot \frac{V_{i}^{-} \cdot V_{i}^{\text {nom }}}{V_{i}^{\text {nom }}-V_{i}^{-}} .
$$

The penalty associated with a positive shift, $\rho_{i}^{+}$, is computed similarly.

A linear expression for the penalty is obtained by introducing a pair of positive variables $\left(\Delta_{T, i}^{-}, \Delta_{T, i}^{+}\right)$together with the constraint $T_{i}^{f}-T=\Delta_{T, i}^{+}-\Delta_{T, i}^{-}$. The time shift is then penalized by adding the terms $\rho_{i}^{+} \Delta_{T, i}^{+}+\rho_{i}^{-} \Delta_{T, i}^{-}$to the objective function.

\subsection{Summary of the model}

For clarity, the sets of variables and the model are summarized below. The complete model is referred to as SPACE in the remainder of this article. We emphasize that the objective function and the constraints of SPACE are linear in the variables.

Variables

$\forall\left(A_{i}, n_{k}, n_{p}\right) \in \mathcal{E}:\{$ decision variables for maneuvers $\}$

$d_{i}^{k p} \geq 0, t_{i}^{k p} \in \mathbb{R}$ : length of speed maneuver, and resulting time shift

$\chi_{i}^{k p} \in(-\pi, \pi], l_{i}^{k p} \geq 0$ : heading and length of heading maneuver

$\epsilon_{i, n}^{k p} \in\{0,1\}, l_{i, n}^{k p} \geq 0$ : choice of the heading and associated length $\left(\forall n \in\left\{1, \ldots, N_{\chi}\right\}\right)$

$z_{i}^{k p} \geq 0$ : fuel consumption

$\forall\left(A_{i}, A_{j}, n_{k}\right) \in C_{C} \cup C_{F}:\{$ decision variables for conflicts $\}$

$\delta_{i j} \in\{0,1\}$ : choice of a configuration to solve the conflict

$\forall\left(A_{i}, n_{k}\right) \in \mathcal{N}$ : $\{$ intermediary variables describing the computed conflict graph $\}$

$T_{i}^{k} \geq 0, \Delta_{i}^{k} \in \mathbb{R}$ : time and lateral shift over $O_{i}^{k}$ (for $n_{k}$ on the trajectory of $A_{i}$ )

$D_{i}^{k p} \geq 0$ : length traveled from $O_{i}^{k}$ to $O_{i}^{p}$ (for $\left.\left(A_{i}, n_{k}, n_{p}\right) \in \mathcal{E}\right)$ 
$\Delta_{T, i}^{-} \geq 0, \Delta_{T, i}^{+} \geq 0$ : negative and positive time shift at the final node

$s_{i}^{k-} \geq 0, s_{i}^{k+} \geq 0$ : extremities of the interval centered on $O_{i}^{k}$ on which the speed vector of $A_{i}$ must be constant (for $n_{k}$ on the trajectory of $A_{i}$ )

\section{Complete model}

$\min \sum_{\left(A_{i}, n_{k}, n_{p}\right) \in \mathcal{E}} z_{i}^{k p}+\sum_{A_{i} \in \mathcal{A}}\left[\rho_{i}^{+} \Delta_{T, i}^{+}+\rho_{i}^{-} \Delta_{T, i}^{-}\right], \quad$ subject to:

$\forall A_{i} \in \mathcal{A}, n_{f}$ the final node of $A_{i}: \Delta_{i}^{f}=0$, and $T_{i}^{f}-T=\Delta_{T, i}^{+}-\Delta_{T, i}^{-}$,

$\forall\left(A_{i}, n_{k}, n_{p}\right) \in \mathcal{E}, \forall n \in\left\{1, \ldots, N_{Z}\right\}: z_{i}^{k p} \geq \alpha_{i}^{n} t_{i}^{k p}+\beta_{i}^{n} d_{i}^{k p}+\left(D_{i}^{k p}-d_{i}^{k p}\right) C_{d, i}\left(V_{i}^{\mathrm{nom}}\right)$,

$\{B T$ recovery $\}$

$\forall\left(A_{i}, n_{k}, n_{p}\right) \in \mathcal{E}:$

\{fuel consumption\}

$\chi_{i}^{k p}=\sum_{n=1}^{N_{\chi}} \epsilon_{i, n}^{k p} \chi_{n}, \quad \sum_{n=1}^{N_{\chi}} \epsilon_{i, n}^{k p} \leq 1, \quad l_{i}^{k p}=\sum_{n=1}^{N_{\chi}} l_{i, n}^{k p}$,

$l_{i, n}^{k p} \leq\left(\frac{\hat{D}_{i}^{k p}}{\cos \chi_{n}}-2 \frac{V_{i}^{\text {nom }}}{\omega^{\max }} \sin \left|\chi_{n}\right|\right) \cdot \epsilon_{i, n}^{k p} \forall n \in\left\{1, \ldots, N_{\chi}\right\}$,

$\forall\left(A_{i}, n_{k}, n_{p}\right) \in \mathcal{E}:$

\{choice of the heading\}

$\frac{d_{i}^{k p}}{V_{i}^{\max }}-\frac{d_{i}^{k p}}{V_{i}^{\text {nom }}}+\frac{\left(V_{i}^{\text {nom }}-V_{i}^{\max }\right)^{2}}{U_{i}^{\max } V_{i}^{\max }} \leq t_{i}^{k p} \leq \frac{d_{i}^{k p}}{V_{i}^{\min }}-\frac{d_{i}^{k p}}{V_{i}^{\text {nom }}}-\frac{\left(V_{i}^{\text {nom }}-V_{i}^{\min }\right)^{2}}{U_{i}^{\max } V_{i}^{\min }}$

$s_{i}^{k^{+}} \geq \frac{\Delta_{i}^{k}}{\sin \theta_{i j}}-\frac{\Delta_{j}^{k}}{\tan \theta_{i j}}+\frac{D}{\sin \left|\theta_{i j}\right|}, \forall\left(A_{i}, A_{j}, n_{k}\right) \in C_{C}$

$s_{i}^{p^{-}} \leq \frac{\Delta_{i}^{p}}{\sin \theta_{i j}}-\frac{\Delta_{j}^{p}}{\tan \theta_{i j}}-\frac{D}{\sin \left|\theta_{i j}\right|}, \forall\left(A_{i}, A_{j}, n_{p}\right) \in C_{C}$

$d_{i}^{k p} \leq D_{i}^{k p}+s_{i}^{p-}-s_{i}^{k+}$

$l_{i, n}^{k p} \cos \chi_{n} \leq \hat{D}_{i}^{k p}+s_{i}^{k-}-s_{i}^{p+}-2 \epsilon_{i, n}^{k p} \frac{V_{i}^{\text {nom }}}{\omega^{\max }}\left(\left|\sin \chi_{n}\right|\right), \quad \forall n \in\left\{1, \ldots, N_{\chi}\right\}$

$\forall\left(A_{i}, n_{k}, n_{p}\right) \in \mathcal{E}$ :

$\{$ states at the nodes\}

$D_{i}^{k p}=\hat{D}_{i}^{k p}+\sum_{n=1}^{N_{\chi}}\left[2 \epsilon_{i, n}^{k p} \times \frac{V_{i}^{\text {nom }}}{\omega^{\max }}\left(\left|\chi_{n}\right|-\left|\sin \chi_{n}\right|\right)+l_{i, n}^{k p}\left(1-\cos \chi_{n}\right)\right]$

$\Delta_{i}^{p}=\Delta_{i}^{k}+\sum_{n=1}^{N_{\chi}}\left[2 \epsilon_{i, n}^{k p} \times \operatorname{sign}\left(\chi_{n}\right) \frac{V_{i}^{\text {nom }}}{\omega^{\max }}\left(1-\cos \chi_{n}\right)+l_{i, n}^{k p} \sin \chi_{n}\right]$

$T_{i}^{p}=T_{i}^{k}+t_{i}^{k p}+\frac{D_{i}^{k p}}{V_{i}^{\text {nom }}}$,

$\forall\left(A_{i}, A_{j}, n_{k}\right) \in C_{C}$ :

$T_{i}^{k}+\frac{1}{V_{i}^{\text {nom }}}\left(\frac{\Delta_{i}^{k}}{\tan \theta_{i j}}-\frac{\Delta_{j}^{k}}{\sin \theta_{i j}}\right)-T_{j}^{k}-\frac{1}{V_{j}^{\text {nom }}}\left(-\frac{\Delta_{j}^{k}}{\tan \theta_{i j}}+\frac{\Delta_{i}^{k}}{\sin \theta_{i j}}\right) \geq T_{i j}^{\min }-M \delta_{i j}$,

$T_{j}^{k}+\frac{1}{V_{j}^{\text {nom }}}\left(-\frac{\Delta_{j}^{k}}{\tan \theta_{i j}}+\frac{\Delta_{i}^{k}}{\sin \theta_{i j}}\right)-T_{i}^{k}-\frac{1}{V_{i}^{\text {nom }}}\left(\frac{\Delta_{i}^{k}}{\tan \theta_{i j}}-\frac{\Delta_{j}^{k}}{\sin \theta_{i j}}\right) \geq T_{i j}^{\min }-M\left(1-\delta_{i j}\right)$

$\forall\left(A_{i}, A_{j}, n_{k}\right) \in C_{T}: T_{i}^{k} \leq T_{j}^{k}-\frac{D}{\min \left(V_{i}^{\min } \cos \chi_{i}^{\max }, V_{j}^{\max }\right)}$,

\{trailing conflicts\}

$\forall\left(A_{i}, A_{j}, n_{k}\right) \in C_{F}: \Delta_{i}^{k}-\Delta_{j}^{k} \geq D-M \cdot \delta_{i j}$ and $\Delta_{j}^{k}-\Delta_{i}^{k} \geq D-M \cdot\left(1-\delta_{i j}\right)$.

$\{$ frontal conflicts $\}$ 


\section{Computational evaluation of the model}

\subsection{Generating a benchmark of artificial instances}

The experimental tests are run on instances implementing the patterns depicted in Figure 7 The grid and roundabout scenarios are the same as in the benchmark described in [17]. The intersection of trails is added to study the impact of the intersection angle in a traffic structure that may be regarded as a special case of the grid. Moreover, organizing the aircraft into trails is an approach that is worth studying in anticipation of denser traffic. The three patterns represent complex situations in which many severe conflicts occur simultaneously. In particular, as the number of aircraft grows, they become much more complex than the most difficult situations that a controller has to deal with in a lifetime. Moreover the geometry of the patterns induces symmetry, which is known to cause difficulties during the solution of MILPs [14]. The purpose of building such a benchmark is to present the algorithm with a diverse set of conflicts, while testing its computational limits.

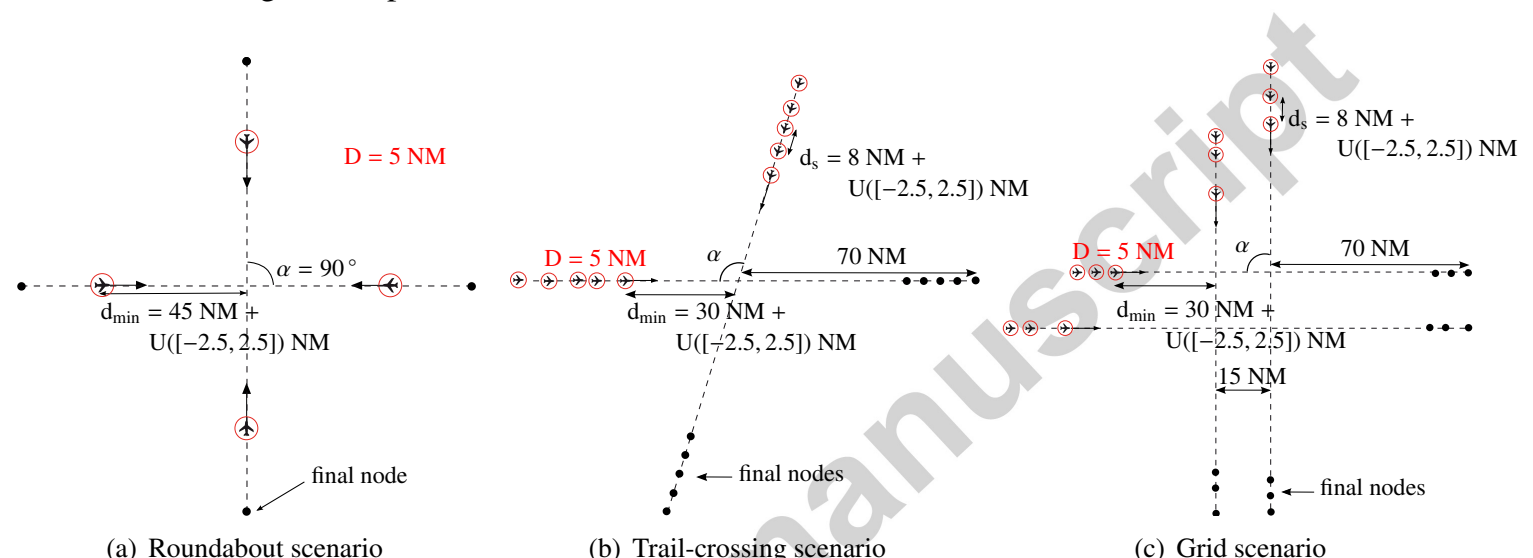

(a) Roundabout scenario

(b) Trail-crossing scenario

(c) Grid scenario

Figure 7: Scenarios used to build the benchmark

For each pattern, we use three to six scenarios to randomly generate 100 instances. The scenarios mostly differ in the number and distribution of the aircraft. They are described in Table 1 . Their characteristics include the total number of aircraft $|\mathcal{A}|$; the number of pairs of aircraft with intersecting trajectories $|C|$; the number of serious conflicts $\left|C_{\triangle}\right|$, i.e., the number of pairs of aircraft that are planned to be separated by less than $D$; and the number of aircraft on each horizontal trail $\left|\mathcal{A}_{x}^{1}\right|$ and $\left|\mathcal{A}_{x}^{2}\right|$ and on each vertical trail $\left|\mathcal{A}_{y}^{1}\right|$ and $\left|\mathcal{A}_{y}^{2}\right|$. When we randomly generate the instances, we set the initial separation between aircraft flying on the same trail to $8+U([-2.5,2.5]) \mathrm{NM}$, where $U([a, b])$ follows a uniform random law on the interval $[a, b]$. This choice is justified by the value of the reference horizontal separation distance, which is equal to $5 \mathrm{NM}$. Even if $-2.5 \mathrm{NM}$ is sampled from the uniform law, the added $8 \mathrm{NM}$ leave a reasonable margin from an initial loss of separation. As indicated in Table 1, the minimum distance between an aircraft and the closest conflict point is also randomly generated, and so is the intersection angle $\alpha$ (see Figure 7) for the trail scenarios. With those random perturbations, the geometric structure is kept but the symmetry is partially broken to ensure that it does not invalidate the results.

For simplicity, the performance profile is assumed to be the same for every aircraft, and it is based on the characteristics found in BADA for an Airbus A320 flying at $33000 \mathrm{ft}$. The bounds on the acceleration, yaw rate, and speed decrease are much more restrictive because they correspond to values that would be accepted by pilots (see [18]) rather than technical limits. The bounds on speed also match the $\pm 6 \%$ subliminal speed changes proposed in the ERASMUS project [8]. The resulting bounds and the other parameters of the model are listed in Table 2, The angles corresponding to $N_{\chi}$ are $\left\{-20^{\circ},-15^{\circ},-10^{\circ},-5^{\circ},-2.5^{\circ}, 2.5^{\circ}, 5^{\circ}, 10^{\circ}, 15^{\circ}, 20^{\circ}\right\}$. The value of $N_{Z}$ is kept low because the resulting approximation is reasonably accurate (see Figure 6), and additional linear segments would imply larger computational times. Finally, the time horizon $T$ is different for each scenario. It corresponds to the flight time from the initial to the final node, and it ranges from twelve to sixteen minutes. 


\begin{tabular}{lcccccccccc}
\hline Type & Scenario & $|\mathcal{A}|$ & $|\mathcal{C}|$ & $\left|\mathcal{C}_{\Delta}\right|$ & $\left|\mathcal{A}_{x}^{1}\right|$ & $\left|\mathcal{A}_{x}^{2}\right|$ & $\left|\mathcal{A}_{y}^{1}\right|$ & $\left|\mathcal{A}_{y}^{2}\right|$ & $\alpha$ & $d_{\text {min }}(\mathrm{NM})$ \\
\hline Trail & $\mathrm{T}-01$ & 2 & 1 & 1 & 1 & - & 1 & - & $U\left(\left[\frac{\pi}{3}, \frac{2 \pi}{3}\right]\right)$ & $42.5+U([-2.5,2.5])$ \\
& $\mathrm{T}-02$ & 4 & 4 & 2 & 2 & - & 2 & - & $U\left(\left[\frac{\pi}{3}, \frac{2 \pi}{3}\right]\right)$ & $32.5+U([-2.5,2.5])$ \\
& $\mathrm{T}-03$ & 6 & 9 & 3 & 3 & - & 3 & - & $U\left(\left[\frac{\pi}{3}, \frac{2 \pi}{3}\right]\right)$ & $27.5+U([-2.5,2.5])$ \\
& $\mathrm{T}-04$ & 8 & 16 & 4 & 4 & - & 4 & - & $U\left(\left[\frac{\pi}{3}, \frac{2 \pi}{3}\right]\right)$ & $27.5+U([-2.5,2.5])$ \\
& $\mathrm{T}-05$ & 10 & 25 & 5 & 5 & - & 5 & - & $U\left(\left[\frac{\pi}{3}, \frac{2 \pi}{3}\right]\right)$ & $27.5+U([-2.5,2.5])$ \\
& $\mathrm{T}-06$ & 12 & 36 & 6 & 6 & - & 6 & - & $U\left(\left[\frac{\pi}{3}, \frac{2 \pi}{3}\right]\right)$ & $27.5+U([-2.5,2.5])$ \\
\hline \multirow{2}{*}{ Grid } & $\mathrm{G}-01$ & 6 & 12 & 5 & 2 & 1 & 2 & 1 & $\frac{\pi}{2}$ & $27.5+U([-2.5,2.5])$ \\
& $\mathrm{G}-02$ & 8 & 16 & 8 & 2 & 2 & 2 & 2 & $\frac{\pi}{2}$ & $27.5+U([-2.5,2.5])$ \\
& $\mathrm{G}-03$ & 10 & 20 & 9 & 3 & 2 & 3 & 2 & $\frac{\pi}{2}$ & $27.5+U([-2.5,2.5])$ \\
& $\mathrm{G}-04$ & 10 & 20 & 9 & 2 & 3 & 2 & 3 & $\frac{\pi}{2}$ & $27.5+U([-2.5,2.5])$ \\
& $\mathrm{G}-05$ & 12 & 36 & 12 & 3 & 3 & 3 & 3 & $\frac{\pi}{2}$ & $27.5+U([-2.5,2.5])$ \\
\hline \multirow{6}{*}{ Roundabout } & $\mathrm{R}-01$ & 4 & 6 & 6 & - & - & - & - & $\frac{\pi}{2}$ & $42.5+U([-2.5,2.5])$ \\
& $\mathrm{R}-02$ & 5 & 10 & 10 & - & - & - & - & $\frac{2 \pi}{5}$ & $42.5+U([-2.5,2.5])$ \\
& $\mathrm{R}-03$ & 6 & 15 & 15 & - & - & - & - & $\frac{\pi}{3}$ & $42.5+U([-2.5,2.5])$ \\
\hline
\end{tabular}

Table 1: Characteristics of the scenarios

\begin{tabular}{cccccccc}
\hline$D$ & $V^{\text {nom }}$ & $V^{\min }$ & $V^{\max }$ & $U^{\max }$ & $\omega^{\max }$ & $N_{\chi}$ & $N_{Z}$ \\
\hline $5 \mathrm{NM}$ & $452 \mathrm{kt}$ & $425 \mathrm{kt}$ & $479 \mathrm{kt}$ & $0.4 \mathrm{kt} . \mathrm{s}^{-1}$ & $0.88^{\circ} \cdot \mathrm{s}^{-1}$ & 6 & 4 \\
\hline
\end{tabular}

Table 2: Optimization and aircraft performance parameters

\subsection{Analysis of the results}

The evaluation of SPACE is based on a comparison with a time-discretized model, denoted TIME, similar to that described in [17]. TIME is an NLP that is solved starting from the optimal solution of an MILP approximating this nonlinear model. We made a few modifications to the model of [17] so that the objective function represents a combination of fuel consumption and delay penalties. TIME was chosen for the comparison because, with the aforementioned adaptation, it accurately models the problem given in Section 2.1, and the authors of [17] reported good computational results.

For each of the fourteen scenarios enumerated in Table 1 , we solve 100 randomly generated instances on a quadcore $2.5 \mathrm{GHz}$ Intel processor with $4 \mathrm{~GB}$ of RAM. We use the Gurobi solver [12] with its default options and $120 \mathrm{~s}$ time limit to solve SPACE and the MILP that approximates TIME. Starting from this initial solution, TIME is then solved with SNOPT ${ }^{2}$ Gurobi implements one of the most efficient branch and cut algorithm for mixed integer linear programming, and SNOPT uses sequential quadratic programming (see [15]). Table 3 summarizes the simulation results averaged over the 100 instances of each scenario. For each model, the table displays the extra fuel consumption ("fuel") and the time shift ("delay") per aircraft caused by the resolution maneuvers. The header "cost" refers to the the ratio of the total extra cost over the cost of the planned trajectories. "cpu" refers to the computational time of the complete solution process: for TIME, this includes both the solutions of the linear approximation and of the NLP. Finally, the last two columns contain the ratios of the computational times and extra costs of TIME to those of SPACE.

The first analysis relates to a piece of information that is not directly displayed. Over the 1400 instances, SPACE always provided a set of conflict-free trajectories, while one failure (on an instance of G-05) was reported when using TIME. Considering that most scenarios are significantly more complex than what can be expected in the current operational context, this indicates that both models should be able to handle most situations arising in practice.

The penultimate column of Table 3 shows that the trajectories of SPACE are less efficient than those of TIME. Overall, the SPACE solutions cost $5 \%$ to $31 \%$ more than the corresponding TIME solutions. This is because the linearization and space discretization shrink the feasible domain of SPACE and produce errors in its estimation of the fuel consumption. Indeed, the original fuel-consumption function is approximated to make SPACE linear, which may result in an optimal solution that would not be optimal if the original criterion were minimized. Moreover, the

\footnotetext{
${ }^{2}$ website : http://www.sbsi-sol-optimize.com/asp/sol product snopt.htm
} 


\begin{tabular}{|c|c|c|c|c|c|c|c|c|c|c|}
\hline & \multicolumn{4}{|c|}{ SPACE } & \multicolumn{4}{|c|}{ TIME } & \multicolumn{2}{|c|}{ TIME/SPACE } \\
\hline & fuel (kg) & delay (s) & $\operatorname{cost}(\% 0)$ & cpu (s) & fuel $(\mathrm{kg})$ & delay (s) & extra $(\% 0)$ & cpu (s) & $\operatorname{cost}$ & cpu \\
\hline F-01 & 0.8 & 0.0 & $1.2 \%$ & 1.0 & 1.0 & 0.0 & $1.5 \%$ & 0.0 & 0.79 & 24.9 \\
\hline F-02 & 1.2 & 0.1 & $1.9 \%$ & 2.5 & 1.3 & 0.2 & $2.2 \%$ & 0.2 & 0.89 & 13.5 \\
\hline F-03 & 1.3 & 0.1 & $2.1 \%$ & 7.8 & 1.4 & 0.2 & $2.4 \%$ & 0.5 & 0.88 & 14.3 \\
\hline F-04 & 1.2 & 0.1 & $1.9 \%$ & 32.7 & 1.4 & 0.2 & $2.2 \%$ & 1.4 & 0.86 & 22.7 \\
\hline F-05 & 1.2 & 0.1 & $1.8 \%$ & 92.8 & 1.3 & 0.2 & $2.1 \%$ & 2.9 & 0.88 & 32.0 \\
\hline F-06 & 1.5 & 0.1 & $2.3 \%$ & 142.2 & 1.7 & 0.1 & $2.5 \%$ & 6.5 & 0.91 & 21.8 \\
\hline G-01 & 1.1 & 0.0 & $1.5 \%$ & 17.8 & 1.3 & 0.1 & $1.9 \%$ & 0.7 & 0.81 & 27.4 \\
\hline G-02 & 1.1 & 0.1 & $1.5 \%$ & 106.1 & 1.5 & 0.4 & $2.0 \%$ & 2.1 & 0.78 & 51.3 \\
\hline G-03 & 1.2 & 0.1 & $1.6 \%$ & 127.4 & 1.6 & 0.4 & $2.1 \%$ & 4.6 & 0.76 & 27.8 \\
\hline G-04 & 1.2 & 0.1 & $1.7 \%$ & 126.9 & 1.6 & 0.5 & $2.2 \%$ & 4.6 & 0.78 & 27.6 \\
\hline G-05 & 1.5 & 0.3 & $2.0 \%$ & 148.4 & 1.6 & 0.6 & $2.1 \%$ & 8.3 & 0.95 & 18.0 \\
\hline R-01 & 1.4 & 0.1 & $2.2 \%$ & 14.4 & 1.7 & 0.2 & $2.7 \%$ & 0.5 & 0.83 & 26.3 \\
\hline $\mathrm{R}-02$ & 1.9 & 0.2 & $3.0 \%$ & 107.7 & 2.3 & 0.9 & $3.5 \%$ & 1.0 & 0.84 & 107.5 \\
\hline R-03 & 2.5 & 0.3 & $3.8 \%$ & 124.4 & 2.8 & 1.1 & $4.3 \%$ & 3.7 & 0.89 & 33.2 \\
\hline
\end{tabular}

Table 3: Compared results of TIME and SPACE

linearization of the constraints, combined with the choice of space discretization, is achieved by important restrictions on the maneuvers. Fixed patterns are imposed for the speed and heading changes; the set of allowed heading changes is discretized; and the speed vectors are constrained to be constant around the conflict points. These measures reduce the flexibility in the design of avoidance maneuvers, so the domain of feasible trajectories is smaller than that for the NLP. It is however important to note that for both models, the maneuvers are incredibly cheap. It never takes more than $0.43 \%$ of the initially planned cost to maintain separation in complex situations and to recover the BTs after the maneuvers. In practical terms, this represents delays smaller than two seconds and an extra fuel consumption that would cost less than $3 \$$ per aircraft (based on the average 2014 price of kerosene). Moreover, the patterns may actually be a positive argument for a practical implementation of the solver as a decision-aid tool. They lead to maneuvers that have geometric and conceptual features that should be easy for human operators to handle.

The last point corresponds to the initial motivation for this work. Complete and realistic time-discretized models may be computationally challenging, as is shown by the average solution times of TIME in Table 3 . On the other hand, finding the optimum of SPACE never takes more than a few seconds, even for the instances of G- 05 which involve twelve aircraft engaged in twelve serious simultaneous conflicts and thirty-six potential conflicts. This represents an improvement by a factor ranging from 13 to 107. The harder task in the solution process of TIME is to find a good feasible solution, which is done by solving the MILP approaching TIME, denoted TMILP in the rest of the article.

For a more specific discussion on the performance of the two models, we provide the dimensions of TMILP and SPACE, together with detailed statistics on their solution process in Table 4. For each MILP, the table displays the number of variables and constraints ("nvars" and "ncons"), the number of separation constraints ("nsep"), the number of branch-and-bound nodes explored during the solution ("nodes"), the computational time in seconds until the optimal solution is found or the time limit reached ("cpu") and the relative optimal gap, i.e., the gap between the best lower and upper bounds of the optimal cost when Gurobi is stopped ("gap"). If the optimal solution is found, the optimality gap takes a very small value (typically $\leq 10^{-6}$ ). Otherwise, it is a valid measure of the remaining computational burden that is required to find the optimal solution and prove the optimality. All the solutions of SPACE were proved to be optimal, so this column is displayed for TMILP only.

For most grid and roundabout instances and for the instances of the two most complex trail scenarios, the solution of TMILP had to be stopped before optimality. For each type of scenarios, the column "gap" of Table 4 shows that the optimality gap increases with the complexity of the scenarios, and reaches $50 \%$ to $72 \%$. This suggests that the "cpu" values strongly underestimate the computational time to optimality for the instances with high optimality gaps. Moreover, TMILP even fails to find a feasible solution for four instances of G-05, which led to the failure of TIME on the unsolved instance of this scenario.This confirms the necessity to solve TMILP before TIME: without a feasible 


\begin{tabular}{|c|c|c|c|c|c|c|c|c|c|c|c|}
\hline & \multicolumn{6}{|c|}{ TIME } & \multicolumn{5}{|c|}{ SPACE } \\
\hline & nvars & ncons & nsep & nodes & cpu (s) & gap & nvars & ncons & nsep & nodes & cpu (s) \\
\hline F-01 & 446 & 638 & 40 & 43 & 0.2 & $0.0 \%$ & 188 & 170 & 2 & 13 & 0.0 \\
\hline $\mathrm{F}-02$ & 1021 & 1453 & 160 & 570 & 0.7 & $0.0 \%$ & 482 & 432 & 8 & 231 & 0.2 \\
\hline F-03 & 1726 & 2442 & 360 & 3944 & 4.3 & $0.0 \%$ & 882 & 786 & 18 & 793 & 0.5 \\
\hline F-04 & 2561 & 3606 & 640 & 21453 & 26.6 & $0.6 \%$ & 1391 & 1237 & 32 & 1786 & 1.4 \\
\hline F-05 & 3526 & 4942 & 1000 & 42301 & 82.2 & $21.2 \%$ & 2007 & 1783 & 50 & 2485 & 2.9 \\
\hline F-06 & 4621 & 6460 & 1440 & 26323 & 120.0 & $67.6 \%$ & 2724 & 2414 & 72 & 5969 & 6.5 \\
\hline G-01 & 1720 & 2418 & 480 & 20821 & 13.7 & $0.0 \%$ & 970 & 866 & 24 & 740 & 0.7 \\
\hline G-02 & 2553 & 3561 & 640 & 72629 & 98.0 & $25.7 \%$ & 1388 & 1228 & 32 & 1868 & 2.1 \\
\hline G-03 & 3516 & 4870 & 800 & 50876 & 113.2 & $38.6 \%$ & 1917 & 1684 & 40 & 2868 & 4.6 \\
\hline G-04 & 3516 & 4879 & 800 & 52053 & 112.9 & $43.0 \%$ & 1914 & 1684 & 40 & 2871 & 4.6 \\
\hline G-05 & 4609 & 6359 & 1440 & 24822 & 120.2 & $71.9 \%$ & 2460 & 2149 & 72 & 4206 & 8.3 \\
\hline R-01 & 1147 & 1583 & 240 & 23773 & 12.1 & $1.1 \%$ & 572 & 516 & 12 & 782 & 0.5 \\
\hline R-02 & 1604 & 2219 & 400 & 121403 & 104.7 & $35.4 \%$ & 530 & 455 & 20 & 2258 & 1.0 \\
\hline R-03 & 2118 & 2905 & 600 & 76104 & 118.9 & $50.3 \%$ & 906 & 798 & 30 & 4200 & 3.7 \\
\hline
\end{tabular}

Table 4: Dimensions and computational performances of the MILPs

starting point, solving the NLP proves to be difficult.

The radical difference in the performances of the two models is mostly due to the number of disjunctive separation constraints, because they directly impact the size of the exploration tree when solving an MILP. With SPACE, one binary choice is required for each potential conflict. TMILP is based on time discretization, so the separation constraints must be satisfied at each sampling time step, and they correspond to a forbidden disk. If a square surface is taken as a linear approximation of the disk, the separation constraint is the disjunction of four constraints corresponding to the four sides of the square. Hence, in TMILP one side of the four needs to be chosen at each time step of the time horizon. The columns "nsep" and "nodes" of Table 4 show how the number of disjunctive constraints impacts the number of nodes explored in the branch-and-bound tree. For TMILP, the variations in the number of nodes are only relevant if the optimal gap is close to zero, because optimality is seldom reached for the other scenarios. Despites this, it is possible to focus on the first four trail scenarios to observe that the increase in the number of nodes approximately fits an exponential curve. Solving SPACE is also a combinatorial decision problem, but the number of disjunctive constraints in SPACE is much smaller than in TMILP. As a consequence, the number of branch-and bound nodes explored during the solution of SPACE remains under control for the tested instances. For the same reason, the dimensions of SPACE and the related solution time will be much less sensitive to an increase in the number of conflicts than those of TMILP.

The computational speed of SPACE is its greatest asset, because ATC has a strong need for reactivity. All the instances were solved in a few seconds although the benchmark includes scenarios that are more complex than the situations occurring in practice. As a consequence we could implement a near real-time device that is capable of proposing deconflicted trajectories. Moreover, since SPACE is an MILP, the optimization guarantees that such a set of trajectories will be found if the model is feasible. Otherwise, it is reasonable to declare that the situation is too complex to be treated via standard procedures. In this case, emergency maneuvers involving, for instance, altitude changes or large deviations from the BT should be considered. This means that the conflict solver could also act as a warning system.

\section{Conclusion}

We have discussed the design of aircraft trajectories that are separated by a reference distance on a given time interval. The motion of the aircraft is assumed to be planar, according to a continuous speed function constrained by limits on the velocity, acceleration, and yaw rate. Finally, the trajectories should recover a contractual business trajectory after the maneuvers and minimize a criterion combining fuel consumption and delay penalties. 
Our approach models the problem with a finite number of constraints and variables through a spatial discretization. Instead of satisfying the constraints at a given set of time steps, the model focuses on a small number of important points of the BT, including the initial and final positions and the conflict points. The motivation is that the resulting model should involve a limited number of variables and constraints and thus be solved in a reasonable computational time.

Our main contribution is the development of a space-discretized MILP, SPACE, allowing both velocity and heading maneuvers, whereas existing MILPs based on this discretization do not consider heading maneuvers. This contribution relies on two restrictions on the trajectories: the maneuvers must belong to a combination of two patterns corresponding to trapezoidal changes of speed and heading, and the speed vectors must be constant around the conflict points. With these restrictions, we can show that heading maneuvers are equivalent to time shifts with respect to the BT, and the minimum time separation can be computed before the optimization, which enables us to model the problem with linear constraints. Another important feature is that the linear objective function reflects a classical indicator of economic efficiency, i.e., a combination of fuel consumption and delay.

We performed tests on a benchmark with 1400 randomly generated instances implementing fourteen different scenarios involving up to twelve aircraft engaged in twelve simultaneous serious conflicts. We compared the performance of SPACE with that of an NLP based on time discretization previously developed in [17], TIME. The results demonstrate that SPACE is perfectly able to handle complex situations: all the instances were solved. The restrictions on the maneuvers led to a fuel consumption $50 \%$ to $100 \%$ higher than that for TIME, but it never reached more than $5 \mathrm{~kg}$ per aircraft, which is surprisingly small for such complex situations. One could also argue that the trapezoidal patterns actually provide a standard procedure that human operators will find easier to handle than unconstrained maneuvers. Finally, the computational tests confirmed the great potential of space discretization. Solving TIME takes more than $120 \mathrm{~s}$ for the most complex instances, but it never took more than a few seconds to solve SPACE. As a consequence, SPACE could be seriously considered as a real-time decision-aid system for conflict resolution. It guarantees that a feasible solution will be found when deconflicted trajectories satisfying the aforementioned restrictions exist. Even if none exist, it provides valuable information since the corresponding situations would certainly require an emergency procedure to be applied.

In future research, we will perform a more thorough study of the implementation potential of the conflict solver. The first step is to gather a sufficiently large number of complex instances based on historical or simulated traffic data. Clearly, the artificial instances used in our experiments allow us to test complex situations, but they do not capture all the issues that may arise in an operational context. Conflicts between aircraft whose altitudes are changing should be taken into account. Uncertainties may also be included in the data to represent, for instance, wind variations or a possible lack of accuracy in the achievement of maneuvers. Since modern flight management systems usually perform very well in maintaining a target heading, the disruptions could be represented by a random error on velocity, as described in [1]. A new MILP could then be derived from SPACE to generate robust separation maneuvers by adding a disruption term to the minimum time separation $T_{i j}^{\min }$.

\section{Acknowledgments}

The author would like to thank Jean-Loup Farges, Thomas Chaboud, and Gérard Verfaillie from the Toulouse center of ONERA, the French Aerospace Lab, for their scientific and moral support and writing assistance. The comments and proofreading of Thibault Lehouillier, GERAD, Canada, were also valuable.

The research described here was funded by ONERA, the French Aerospace Lab.

\section{References}

[1] Alliot, J.-M., Durand, N., 2011. A mathematical analysis of the influence of wind uncertainty on mtcd efficiency. Revue de l'IFATCA, The controller.

[2] Alonso-Ayuso, A., Escudero, L., Olaso, P., Pizarro, C., 2011. Conflict avoidance: 0-1 linear models for conflict detection \& resolution. TOP, Springer, $1-20$.

[3] Alonso-Ayuso, A., Escudero, L. F., Martín-Campo, F., Mladenović, N., 2014. A VNS metaheuristic for solving the aircraft conflict detection and resolution problem by performing turn changes. Journal of Global Optimization, 1-14.

[4] Alonso-Ayuso, A., Escudero, L. F., Martín-Campo, F. J., 2012. A mixed 0-1 nonlinear optimization model and algorithmic approach for the collision avoidance in ATM: Velocity changes through a time horizon. Computers \& Operations Research 39 (12), 3136-3146. 
[5] Bicchi, A., Pallottino, L., 2000. On optimal cooperative conflict resolution for air traffic management systems. IEEE Transactions on Intelligent Transportation Systems 1 (4), 221-231.

[6] Borrelli, F., Subramanian, D., Raghunathan, A. U., Biegler, L. T., 2006. MILP and NLP techniques for centralized trajectory planning of multiple unmanned air vehicles. In: American Control Conference. Vol. 1-12. pp. 5763-5768.

[7] Cafieri, S., Durand, N., 2013. Aircraft deconfliction with speed regulation: New models from mixed-integer optimization. Journal of Global Optimization 58 (4), 613-629.

[8] Drogoul, F., Averty, P., Weber, R., 2009. ERASMUS strategic deconfliction to benefit SESAR. In: Eighth USA/Europe Air Traffic Management Research and Development Seminar.

[9] Durand, N., Alliot, J.-M., 2009. Ant colony optimization for air traffic conflict resolution. In: USA/Europe Air Traffic Management Research and Development Seminar. ATM Seminar, http://www.atmseminar.org.

[10] Earl, M. G., D'Andrea, R., 2007. Multi-vehicle cooperative control using mixed integer linear programming. In: Cooperative Control of Distributed Multi-Agent Systems. John Wiley \& Sons, Ltd., Ch. 10, pp. 231-259.

[11] Frese, C., Beyerer, J., 2010. Planning cooperative motions of cognitive automobiles using tree search algorithms. In: Dillmann, R., Beyerer, J., Hanebeck, U., Schultz, T. (Eds.), KI 2010: Advances in Artificial Intelligence. Vol. 6359 of Lecture Notes in Computer Science. Springer Berlin/Heidelberg, pp. 91-98.

[12] Gurobi Optimization, I., 2012. Gurobi optimizer reference manual. URL http://www.gurobi.com

[13] Lehouillier, T., Allignol, C., Omer, J., Soumis, F., 2014. Interactions between operations and planning in air traffic control. In: Submitted to the International Conference on Research in Air Transportation (ICRAT).

[14] Margot, F., 2010. Symmetry in integer linear programming. In: 50 Years of Integer Programming 1958-2008. Springer Berlin Heidelberg, pp. 647-686.

[15] Nocedal, J., Wright, S., 2006. Numerical optimization, $2^{\text {nd }}$ edition Edition. Springer series in operations research. Springer.

[16] Nuic, A., 2011. User manual for the Base of Aircraft Data (BADA). Tech. Rep. 11/03/08-08, Eurocontrol URL http://www.eurocontrol.int/eec/public/standard_page/proj_BADA.html

[17] Omer, J., Farges, J.-L., 2013. Hybridization of nonlinear and mixed-integer linear programming for aircraft separation with trajectory recovery. IEEE Transactions on Intelligent Transportation Systems 14 (3), 1218-1230.

[18] Paielli, R. A., 2003. Modeling maneuver dynamics in air traffic conflict resolution. Journal of Guidance, Control, and Dynamics 26, 407-415.

[19] Pallottino, L., Feron, E., Bicchi, A., 2002. Conflict resolution problems for air traffic management systems solved with mixed integer programming. IEEE Transactions on Intelligent Transportation Systems 3, $3-11$

[20] Raghunathan, A. U., Gopal, V., Subramanian, D., Biegler, L. T., Samad, T., 2004. Dynamic optimization strategies for three-dimensional conflict resolution of multiple aircraft. Journal of Guidance, Control, and Dynamics 27 (4), 586-594.

[21] Rey, D., Rapine, C., Fondacci, R., Faouzi, N.-E. E., 2012. Minimization of potential air conflicts through speed regulation. In: Transportation Research Record: Journal of the Transportation Research Board. No. 2300. pp. 59-67.

[22] Richards, A., How, J., 2002. Aircraft trajectory planning with collision avoidance using mixed integer linear programming. In: American Control Conference. Vol. 3. pp. 1936-1941.

[23] Schouwenaars, T., 2006. Safe trajectory planning of autonomous vehicles. Ph.D. thesis, Massachusetts Institute of Technology, Department of Aeronautics and Astronautics, Cambridge MA.

[24] SESAR Joint Undertaking, 2012. European ATM master plan, edition 2. Tech. rep.

[25] STATFOR, E., 2013. Eurocontrol long-term forecast: IFR flight movements 2013-2035. Tech. rep., Eurocontrol.

[26] Vela, A., Solak, S., Clarke, J., Singhose, W., Barnes, E., Johnson, E., 2010. Near real-time fuel-optimal en route conflict resolution. IEEE Transactions on Intelligent Transportation Systems 11, 826-837.

[27] Vela, A., Solak, S., Singhose, W., Clarke, J.-P., 2009. A mixed integer program for flight-level assignment and speed control for conflict resolution. In: Proceedings of the 48th IEEE Conference on Decision and Control. pp. 5219-5226. 